\title{
A VARIATIONAL INEQUALITY REFORMULATION OF A CONGESTED TRANSIT ASSIGNMENT MODEL BY COMINETTI, CORREA, CEPEDA AND FLORIAN ${ }^{1}$.
}

\author{
by \\ Esteve Codina ${ }^{\dagger}$ \\ ${ }^{\dagger}$ Statistics and Operations Research Department \\ Universitat Politècnica de Catalunya \\ Campus Nord, Building C5, Office 216 \\ C/ Jordi Girona, 1-3, Barcelona 08034, Spain
}

This is the peer reviewed version of the following article: Codina, E. "A variational inequality reformulation of a congested transit assignment model by Cominetti, Correa, Cepeda and Florian", which has been published in final form at Trans. Sci. (2012) 47 (2), 231-246. DOI 10.1287/trsc.1120.0427

\begin{abstract}
In the paper by Cominetti and Correa (2001) [Common-lines and passenger assignment in congested transit networks. Transportation Science 35 (3), pp 250-267], an extension to the common-lines problem for general multidestination networks under congestion is analyzed. Their transit equilibrium assignment model allows for a full representation of congestion effects caused by the variation of effective frequencies experienced by passengers at transit stops. This model is the first to address these characteristics consistently with the concept of strategies. In a subsequent paper by Cepeda et al. (2006) [Cepeda, M., Cominetti, and R. Florian, M. (2006) A frequency-based assignment model for congested transit networks with strict capacity constraints: characterization and computation of equilibria. Trans. Res B 40, 437-459], the computation of equilibrium is performed heuristically by the minimization of a gap function, using the method of successive averages. In this paper, a reformulation of this congested transit equilibrium assignment model is performed, demonstrating that the problem can be expressed as an equivalent variational inequality. The case of strictly capacitated transit networks is explored under the scope of this new reformulation, and new, broader conditions for the existence of solutions to this congested transit assignment model are determined.
\end{abstract}

\section{Introduction}

The development of transit assignment models of increasing complexity has been driven mainly by the inclusion of congestion effects caused by the complexity of passengers' behavior and limitations in the physical capacity of transportation vehicles. However, public transportation is seen as an appropriate way to mitigate the increase in demand for transportation and its associated environmental effects. Despite this observation, the development of congested transit assignment models has previously received less attention when compared, for example, with traffic assignment models. Thus, the number of contributions in the field of transit assignment can be considered more limited than in the case of traffic assignment models and in the applications that require them, such as matrix adjustment methods and continuous network design problems. One explanation for these limitations stems from the compact formulation of user equilibrium using variational inequalities (VIs) for the traffic assignment problem, as shown in Smith (1979). This VI formulation, has not been achieved for previous transit assignment models but in their simpler instances. Advantages for using VI formulations are also discussed in Marcotte (1995).

A brief description of the evolution of transit assignment models will be presented at this point. The paper by Chriqui and Robillard (1975) introduced the notion that passengers can select a subset of attractive lines and board the first vehicle arriving at a stop to minimize the expected sum of waiting

\footnotetext{
${ }^{1}$ Research supported under Spanish Research Project TRA2005-09068-C03-02
} 
plus travel times. An extension of this idea was explored by Spiess (1984) and by Spiess and Florian (1989), who introduced the notion of strategy as the choice of an attractive set of lines at each stop. The resulting model seeks to minimize the expected value of the total travel time. Nguyen and Pallotino (1988) interpreted a strategy as a hyperpath, i.e., an acyclic directed graph that leads to a destination. In these models, the only aspect of congestion under consideration is the association of discomfort functions with transit line segments, enabling the resulting equilibrium model to be formulated as a convex programming problem. In these models, waiting times are underestimated because it is not assumed that passengers will board the first vehicle after their arrival at the stop, but will probably have to wait for subsequent vehicles because of congestion.

The first transit network model that takes into account limitations in line capacity can be credited to De Cea and Fernández (1993), who concentrate on the congestion effects at transfer nodes by means of BPR -like functions. Both existing passenger flows on the lines prior to boarding and boarding flows are taken into account. Nevertheless, this model allows line capacity to be exceeded, and the formulation does not take into consideration any queuing theory at stops.

Cominetti and Correa (2001) develop a general frequency-based transit equilibrium model with arbitrary functions that model travel times and effective frequencies. These authors analyzed the commonlines problem presented by Chriqui and Robillard and formulated it as a fixed-point problem without proposing an algorithm to solve it, although they proved that solutions do exist. As a continuation of this work, Cepeda et al. (2006) showed that the model of Cominetti and Correa (2001) can be formulated equivalently as the minimization of a non-differentiable and non-convex gap function. They solve the problem heuristically using an adaptation of the method of successive averages and show the computational viability of their approach on large transit networks. In this paper, we refer to this model as the Cominetti-Correa-Cepeda-Florian model (C3F). The main contribution of this paper is to show that the $\mathrm{C} 3 \mathrm{~F}$ model can be reformulated as a VI problem, thus expressing in a very compact and convenient form the congested transit equilibrium assignment based on strategies for its analysis and algorithmic treatment. From the perspective of the variational inequality formulation stated in the paper for the C3F model, it is shown how the MSA heuristic method used by Cepeda et al. (2006) can be interpreted as a method to find a solution of a fixed- point inclusion. This reformulation as a fixed-point inclusion problem also arises directly from the VI reformulation conducted in this paper. The algorithmic consequences of the new V.I. formulation are examined in a subsequent paper. The case of transit models with high levels of congestion is analyzed when effective frequency functions (efff) are present that impose sharp implicit capacity constraints. For these problems, it is shown that solutions of the C3F model are strictly under capacity bounds.

The paper is organized as follows. Section 2 presents basic notations and definitions. Section 3 summarizes the current state of development of a congested transit assignment model consistent with the concept of strategies as analyzed by Cominetti and Correa (2001), and particular attention is paid to the contribution by Cepeda et al. (2006). In section 4, it is rigorously proved that the minimization of the gap function in Cepeda et al. (2006) is equivalent to a generally nonmonotone V.I. problem. In section 5, the question of the existence of solutions to the newly reformulated V.I. is examined for two cases. In the first case, no strict capacity bounds on transit lines are imposed, and efff's are assumed to remain positive in the space of flows, although they may decrease conveniently for high transit volumes. In the second case, the presence of strict capacity bounds imposed when efff's vanish is addressed. For this important case, assumptions ensuring the existence of solutions for the capacitated transit assignment model are developed under the scope of the new V.I. reformulation, and it is shown that, under these assumptions, flows on transit lines are under capacity, avoiding the occurrence of infinite travel times. In both cases, conditions for the existence of solutions of the congested transit assignment model are broader than the conditions shown by Cominetti and Correa (2001). Finally, section 6 presents the main conclusions of the paper.

\section{Basic notation and a network model}

The transit network is represented by means of a directed graph $G=(N, A)$, where $N$ is the set of nodes and $A$ is the set of links. Generic nodes in $N$ will be represented by the symbols $i, j$. A link will usually be represented by the symbol $a$ and explicitly by $(i, j)$ or $\left(i, j_{a}\right)$, depending on the context.

It will be assumed that there exists a subset $C$ of nodes on which trips may originate and/or end. Passenger trips are associated with origin-destination pairs of nodes $(i, d)$, both $i, d \in C$. The number of 
trips from $i$ to $d$ will be denoted by $g_{i}^{d}$. By $W$, the set of active origin-destination pairs $\omega=(i, d)$ on the network is denoted, i.e., those pairs for which some trip exists: $g_{i}^{d}>0$.

$$
W \triangleq\left\{(i, d) \in C \times C \mid g_{i}^{d}>0\right\}
$$

The set of destinations in the network will be denoted by $D$.

$$
D \triangleq\{d \in C \mid \exists(i, d) \in W\}
$$

and the set of origin nodes for a fixed destination $d \in D$ will be denoted by $O(d)$

$$
O(d) \triangleq\{i \in C \mid(i, d) \in W\}
$$

Often, when referring to a given destination $d \in D$, the set of nodes in the network excluding destination $d$ will be denoted by $N_{d}$, i.e., $N_{d}=N \backslash\{d\}$. In general, for a node $i \in N$, the set of emerging links will be denoted by $E(i)$, and the set of incoming links by $I(i)$. The network will be considered in its detailed expanded form, following Spiess (1984) and Spiess and Florian (1989), as shown in figure 1. In this representation, transit stops are associated with a node for which some of the outgoing links will play the role of boarding links to a transit line, and some of the incoming links will play the role of alighting links from a transit line. Each transit line with vehicles halting at the stop will have a single boarding link from the stop and a single alighting link to the stop. Non-boarding or non-alighting links incoming to or outgoing from nodes representing transit stops will model connections to other transportation modes, such as pedestrian or pedestrian connections to other transit stops.

Because the model can be stated as a multi-destination network flow, $v_{a}^{d}$ will denote the flow at link $a \in A$ with destination $d \in D$. Then, the following notation will be used for the various types of vector flows and origin-destination volumes:

- $\mathbf{v}_{i}^{d}=\left(\ldots, v_{a}^{d}, \ldots ; a \in E(i)\right) \in \mathbb{R}_{+}^{|E(i)|}, i \in N, d \in D$ is the vector of flows with destination $d$ at emerging links of node $i$.

- $v_{i}^{d}=\sum_{a \in E(i)} v_{a}^{d}$ is the total inflow through node $i \in N$ with destination $d \in D$. Notice that no confusion may arise between $v_{i}^{d}$ and $v_{a}^{d}$ because indexes are always properly declared in formulas and when mentioned in the text. Furthermore, index $a$ is shorthand for an ordered pair of indexes $(i, j)$, both in $N$.

- $\mathbf{v}^{d}=\left(\ldots, \mathbf{v}_{i}^{d}, \ldots ; i \in N\right) \in \mathbb{R}_{+}^{|A|}, d \in D$.

- $\mathbf{v}=\left(\ldots, \mathbf{v}^{d}, \ldots ; d \in D\right) \in \mathbb{R}_{+}^{|A||D|}$.

- $v=\sum_{d \in D} \mathbf{v}^{d} \in \mathbb{R}_{+}^{|A|}$ is the vector of total flows on links, and $v_{a}=\sum_{d \in D} v_{a}^{d}, a \in A$.

- $\mathrm{g}^{d}=\left(\ldots, g_{i}^{d}, \ldots ; i \in O(d)\right) \in \mathbb{R}_{+}^{|O(d)|}, d \in D$

- $\mathrm{g}=\left(\ldots, \mathrm{g}^{d}, \ldots ; d \in D\right) \in \mathbb{R}_{+}^{|W|}$.

With previous definitions, the feasibility set for the congested transit equilibrium problem can be formulated as:

$$
\mathrm{V} \triangleq \bigotimes_{d \in D} \mathrm{~V}^{d}
$$

where each set $\mathrm{V}^{d}$ is defined as:

$$
\mathrm{V}^{d} \triangleq\left\{\mathbf{v}^{d} \in \mathbb{R}_{+}^{|A|} \mid \sum_{a \in E(i)} v_{a}^{d}-\sum_{a \in I(i)} v_{a}^{d}=g_{i}^{d} i \in N_{d}, \quad \sum_{a \in I(d)} v_{a}^{d}=\sum_{i \in O(d)} g_{i}^{d}, v_{a}^{d}=0, \forall a \in E(d)\right\}, d \in D
$$

In addition, the feasible set $\mathcal{V}$ of total link flows $v$ can be defined as: 


$$
\mathcal{V} \triangleq\left\{v \in \mathbb{R}_{+}^{|A|} \mid v=\sum_{d \in D} \mathbf{v}^{d}, \mathbf{v}^{d} \in \mathrm{V}^{d}\right\}
$$

Boarding links $a$ from stop $i$ are associated with a unique transit line and have associated a nonnegative efff $f_{a}(\cdot): \mathcal{V} \rightarrow \mathbb{R}$ for that line, which will generally depend on the total flows of some neighbor links and on the total flow of link $a$. Effective frequency are the inverse of the expected waiting time at the stop until boarding onto a vehicle of the line. Because of the finite capacity of vehicles, this boarding may not happen on the first arriving vehicle seen by the passenger. This gives rise to a bulk service type of queuing process for passengers at stops. Mean waiting times for a boarding, or the inverse of effective frequencies, will be denoted by $\sigma_{a}(\cdot)=1 / f_{a}(\cdot)$. Thus, the role of efff at boarding links is to model the limitation in the capacity of transit vehicles. Although no predetermined functional form is assumed in either Cominetti and Correa (2001) or Cepeda et al. (2006), it can be assumed that functions $f_{a}(\cdot)$ are finite for flows $v \in \mathcal{V}$ that verify some type of (typically linear) inequality $c_{a}(v)<\bar{c}_{a}$, vanish at flows that verify strict equality $c_{a}(v)=\bar{c}_{a}$, have a maximum value for flows that verify $c_{a}(v)=0$ and remain undefined at flows such that $c_{a}(v)>\bar{c}_{a}$.

Travel times on links will be modeled by general functions $t_{a}(v), a \in A$, which remain bounded on $\mathcal{V}$, i.e., $0 \leq t_{a}(v)<+\infty, \forall v \in \mathcal{V}, a \in A$.

The subset of nodes for which emerging links exist with a bounded from above (finite) efff on $\mathcal{V}$ will be denoted by $\hat{N}$.

$$
\hat{N} \triangleq\left\{i \in N \mid \exists a \in E(i), f_{a}(\cdot)<+\infty\right\}
$$

For simplicity of formulas, the sets $\hat{N}_{d}=\hat{N} \backslash\{d\}, d \in D$ and $\hat{A}=\{a \in A \mid \exists i \in \hat{N}, a \in \hat{E}(i)\}$ will also be used. For nodes $i \in N$, the subset of emerging links with finite effective frequency will be denoted by $\hat{E}(i)$ :

$$
\hat{E}(i) \triangleq \begin{cases}\left\{a \in E(i) \mid f_{a}(\cdot)<+\infty\right\}, & i \in \hat{N} \\ \emptyset, & i \in N \backslash \hat{N}\end{cases}
$$

and the set of boarding links at a stop $i \in \hat{N}$ with positive destination flows will be denoted by:

$$
\hat{E}_{+}^{d}(i, \mathbf{v})=\left\{a \in \hat{E}(i) \mid v_{a}^{d}>0, i \in \hat{N}\right\}
$$

A strategy for passengers at node $i \in \hat{N}$ will be represented simply by a subset of links $\mathcal{E}(i) \subseteq \hat{E}(i)$.

Figure 1

Line segments as well as pedestrian, transfer and non-transit facilities will be represented by links $a \in A$ with either constant or flow-dependent travel time functions $t_{a}(\cdot)$ and infinite frequencies, $f_{a}=+\infty$. The same will apply for links $a \in I(i), i \in \hat{N}$, representing alighting at stops.

For V.I. formulations used in the paper, the following notation will be used. If $C \subseteq \mathbb{R}^{n}$ is a convex set, then for an operator $\Phi: \mathbb{R}^{n} \rightarrow \mathbb{R}^{n}$, a V.I. will be formulated in its classical form, i.e., find $x \in C$ so that $\Phi(x)^{\top}\left(x^{\prime}-x\right) \geq 0, \forall x^{\prime} \in C$. This VI will also be referred to as $\operatorname{VI}(\Phi, C)$, and its solution set by Sol $\operatorname{VI}(\Phi, C)$.

In formulas or expressions describing optimization problems, the Min or Max operator will be subscripted with vectors or variables that play the role of "decision variables" or optimization variables appearing in the body of the problem, with some or all of their sub/superscripts probably suppressed for legibility. In this way, a clear distinction between optimization variables and parameters of the problem can be made. Additionally, when considered convenient, immediately after a constraint, dual variables or multipliers for that constraint may appear after a bar "|". In other words, $v \leq w r, \mid \theta$ indicates that $\theta$ is the dual variable for constraint $v \leq w r$.

\section{A brief summary of the C3F model}

\subsection{The common-lines problem with congestion}

The C3F model can be considered an extension to the case of general multi-destination networks of the so-called common-lines problem (CLP in the following) when effects of congestion are reflected as a drop 
of the line frequencies experienced by passengers. This problem can be summarized as follows. Let us consider a simple network consisting of a single origin node connected to its destination by means of a set of bus lines $\nu=1,2, \ldots, n$ with effective frequencies $f_{\nu}(\cdot)$, which are decreasing functions of the boarding flows $v_{\nu}$, vanishing at a maximum flow or capacity $\bar{v}_{\nu}$. Assume that the trip demand from origin to destination is $x=\sum_{\nu=1}^{n} v_{\nu}$. Assume that $t_{\nu}<+\infty$ is the in-vehicle travel time for line $\nu$ from the origin node to the destination. Then, users choosing to board on a subset of lines (or strategy) $\mathcal{E}$ will experience an average origin-destination travel time given by:

$$
T_{\mathcal{E}}(v)=\frac{1+\sum_{\nu \in \mathcal{E}} t_{\nu} f_{\nu}\left(v_{\nu}\right)}{\sum_{\nu \in \mathcal{E}} f_{\nu}\left(v_{\nu}\right)}
$$

Let $y_{\mathcal{E}}$ denote the flow of users choosing strategy $\mathcal{E}$. If $\mathcal{E}_{\nu} \subseteq \mathcal{P}(\{1, \ldots, n\})$ is the set of strategies containing line $\nu$, then the total flow $v_{\nu}$ of line $\nu$ will be given by:

$$
v_{\nu}=\sum_{\mathcal{E} \in \mathcal{E}_{\nu}} y_{\mathcal{E}} \frac{f_{\nu}\left(v_{\nu}\right)}{\sum_{\nu^{\prime} \in \mathcal{E}} f_{\nu^{\prime}}\left(v_{\nu^{\prime}}\right)}, \quad \nu=1, \ldots, n
$$

A vector $y=\left(\ldots, y_{\mathcal{E}}, \ldots ; \mathcal{E} \in \mathcal{P}(\{1, \ldots, n\})\right)$ of flow strategies determines a unique vector of line flows $\left(\ldots, v_{\nu}, \ldots ; 1 \leq \nu \leq n\right)$, and the concept of an equilibrium flow vector strategy $y^{*}$ is defined by:

$$
\begin{aligned}
& y_{\mathcal{E}}^{*}>0 \Rightarrow T_{\mathcal{E}}\left(v\left(y^{*}\right)\right)=\hat{T}\left(v\left(y^{*}\right)\right) \\
& T_{\mathcal{E}}\left(v\left(y^{*}\right)\right)-\hat{T}\left(v\left(y^{*}\right)\right)>0 \Rightarrow y_{\mathcal{E}}^{*}=0
\end{aligned}
$$

where $\hat{T}(v) \triangleq \operatorname{Min}_{\mathcal{E} \in \mathcal{P}(\{1, \ldots, n\})}\left\{T_{\mathcal{E}}(v)\right\}$ is the origin-destination minimum travel time at equilibrium. The congested common-lines problem is examined in Cominetti and Correa (2001), in which the existence of equilibrium solutions is proved to exist for any $x \in] 0, \sum_{\nu=1}^{n} \bar{v}_{\nu}[$, showing additionally that, in this case, equilibrium solutions are such that $v_{\nu}<\bar{v}_{\nu}$ and that consequently, origin-destination travel times at equilibrium remain bounded. Also in Cominetti and Correa (2001), the equilibrium notion for the congested CLP is extended to the case of general transit networks with multiple origin-destination pairs.

If we consider now the CLP for the case in which some of the lines, for example, lines $n-k$ to $n$, work with infinite frequency and finite in-vehicle travel times, $\hat{T}(v)$ would need to be defined as:

$$
\hat{T}(v) \triangleq \operatorname{Min}\left\{\operatorname{Min}_{\mathcal{E} \in \mathcal{P}(\{1, \ldots, n-k\})}\left\{T_{\mathcal{E}}(v)\right\}, \operatorname{Min}_{n-k+1 \leq \nu \leq n}\left\{t_{\nu}\right\}\right\}
$$

However, the same concept of equilibrium expressed in (12) would be more difficult to define, although in essence, it would also hold.

\subsection{Notion of equilibrium on general multidestination networks. The C3F model}

Let us consider a general transit network with multiple origin-destination pairs. Using definitions in section 2 , let $\mathbf{v}$ be a feasible vector of per-destination flows, and let $v$ be its corresponding feasible flow vector of total flows. For vector $\mathbf{v}$, consider the congested CLP at node $i \in N$ for destination $d \in D$, $\mathrm{CLP}_{i}^{d}(\mathbf{v})$, with inflow $v_{i}^{d}=\sum_{a \in E(i)} v_{a}^{d}$. Assume for $\mathrm{CLP}_{i}^{d}(\mathbf{v})$ constant travel times given by $t_{a}^{d}(v)$ and effective frequency $f_{a}^{d}(\cdot)$ for each link $a \in \hat{E}(i)$ obtained from $f_{a}(\cdot)$ by considering it as a function only of the flow $v_{a}^{d}$ and freezing all other flows.

Let $V_{i}^{d, *}(\mathbf{v})$ be the set of equilibrium per-destination flow solutions of $\operatorname{CLP}_{i}^{d}(\mathbf{v})$, and let

$$
V_{C L P}^{*}(\mathbf{v}) \triangleq \bigotimes_{d \in D} \bigotimes_{i \in \hat{N}_{d}} V_{i}^{d, *}(\mathbf{v})
$$

As a generalization of expression (10), now origin-destination travel times $\tilde{\tau}_{i}^{d}(v),(i, d) \in W$, for the set of CLP's $\operatorname{CLP}_{i}^{d}(\mathbf{v})$, defined by vector flows $\mathbf{v}$, are defined recursively by: 


$$
\tau_{i}^{d}(v)=\left\{\begin{array}{c}
\operatorname{Min}_{\mathcal{E}(i) \subseteq E(i)} \\
0 \quad \text { if } i=d
\end{array}\left\{\frac{1+\sum_{a \in \mathcal{E}(i)}\left(\tau_{j_{a}}^{d}(v)+t_{a}^{d}(v)\right) f_{a}^{d}(v)}{\sum_{a \in \mathcal{E}(i)} f_{a}^{d}(v)}\right\}\right.
$$

Definition 3.1 ( of C3F transit equilibrium ) A feasible flow $\mathbf{v} \in \mathrm{V}$ is a transit network equilibrium iff $\forall d \in D$ and any $i \in \hat{N}, i \neq d$, the flow vectors $\mathbf{v}_{i}^{d}$ are local equilibrium solutions of the local common-lines problem defined by the vector flow $\mathbf{v}$ itself, $\operatorname{CLP}_{i}^{d}(\mathbf{v})$, at $i$ for destination d, i.e.:

$$
\left(\ldots, \mathbf{v}_{i}^{d}, \ldots ; i \in \hat{N}, d \in D\right) \in V_{C L P}^{*}(\mathbf{v}) .
$$

The set of solutions for the previous fixed-point problem (16) will be denoted by $\mathrm{V}^{*}$ on the space of per-destination link flows and by $\mathcal{V}^{*}$ on the space of total flows on links. Cominetti and Correa (2001) prove the existence of solutions to the fixed-point problem stated in (16) under the following assumption:

Assumption 3.2 For any origin-destination pair, there exists at least a path on the expanded network comprised of links with infinite frequency and bounded function costs.

Assumption 3.2 ensures that, no matter how large the origin-destination trip volumes may be, it will be always possible to allocate them on the network without allowing for the infeasibility of the problem, and consequently, origin-destination travel times will be bounded from above. Paths $\gamma$ referred to in assumption 3.2 play the role of unlimited capacity paths. Links in these paths may be transfer links from stop to stop or simply pedestrian links from a realistic representation of an urban area. This is a device frequently used by practitioners when modeling transfers from station to station, and the interactions with the pedestrian network, which operates conjointly with the transit facilities.

Although (16) is a characterization of equilibrium in terms of a fixed-point problem, it was not considered as an operative formulation to solve for solutions by Cepeda et al. (2006). Instead, building on the results of Cominetti and Correa (2001), Cepeda et al. (2006) prove that their general multidestination network equilibrium transit notion is equivalent to the minimization of the following non-convex, nondifferentiable gap function $G_{\mathrm{CCF}}(\mathbf{v})$

$$
G_{\mathrm{CCF}}(\mathbf{v})=\sum_{d \in D}\left[\sum_{a \in A} v_{a}^{d} t_{a}(v)+\sum_{i \in N_{d}} \operatorname{Max}_{a \in E(i)}\left\{\frac{v_{a}^{d}}{f_{a}(v)}\right\}-\sum_{i \in N_{d}} g_{i}^{d} \tau_{i}^{d}(v)\right]
$$

over the feasible set of destination flow vectors $\mathrm{V}$; in other words, solutions of (16) are also global minima of the problem

$$
\operatorname{Min}_{\mathbf{v} \in \mathrm{V}} G_{\mathrm{CCF}}(\mathbf{v})
$$

Then, in Theorem 3.2 in Cepeda et al. (2006), it is proved that the gap function $G_{\text {CCF }}$ vanishes at equilibrium, as does each of its component functions $G_{\mathrm{CCF}}^{d}(\mathbf{v})$; in other words, $G_{\mathrm{CCF}}(\mathbf{v})=\sum_{d \in D} G_{\mathrm{CCF}}^{d}(\mathbf{v})$ and

$$
G_{\mathrm{CCF}}^{d}(\mathbf{v}) \triangleq \sum_{a \in A} v_{a}^{d} t_{a}(v)+\sum_{i \in \hat{N}_{d}} \operatorname{Max}_{a \in \hat{E}(i)}\left\{\frac{v_{a}^{d}}{f_{a}(v)}\right\}-\sum_{i \in N_{d}} g_{i}^{d} \tau_{i}^{d}(v)=0, \mathbf{v} \in \mathrm{V}^{*}, d \in D
$$

For the purposes of this paper, the following theorem from Cepeda et al. (2006) is also of interest.

Theorem 3.3 ( Theorem 3.1 in Cepeda et al. (2006)) $\quad \mathbf{v} \in \mathrm{V}^{*}$ iff $\mathbf{v} \in \mathrm{V}$ and there exist numbers $\alpha_{i}^{d} \geq 0$ such that for all $d \in D$ and $i \neq d$

$$
\frac{v_{a}^{d}}{f_{a}(v)}\left\{\begin{array}{ccc}
=\alpha_{i}^{d} & \text { if } \quad t_{a}(v)+\tilde{\tau}_{j_{a}}^{d}(v)<\tilde{\tau}_{i}^{d}(v), & \text { case a) } \\
\leq \alpha_{i}^{d} & \text { if } \quad t_{a}(v)+\tilde{\tau}_{j_{a}}^{d}(v)=\tau_{i}^{d}(v), & \text { case b) } \\
=0 & \text { if } \quad t_{a}(v)+\tilde{\tau}_{j_{a}}^{d}(v)>\tilde{\tau}_{i}^{d}(v), & \text { case c) }
\end{array}\right.
$$




\section{An equivalent formulation of equilibrium by means of varia- tional inequalities}

The objective of this section is to prove formally that the C3F model exposed previously in section 3 can be equivalently formulated as a VI in a finite dimensional space.

From an intuitive point of view, the role of paths described in assumption 3.2 is to guarantee that part of the demand will be absorbed on these paths if necessary. If it is assumed that solution flows will be bounded, then travel time on these paths cannot be $+\infty$, in which case, it seems a reasonable conjecture that efff's $f_{a}(\cdot)$ at C3F equilibrium flows will always remain strictly positive: $f_{a}\left(v^{*}\right)>0, a \in \hat{E}(i), i \in$ $\hat{N}$. The reformulation as a V.I. of the C3F model will be performed in this section under the scope of assumption 3.2. However, this does not imply that the resulting V.I. is only valid under assumption 3.2. In section 5, where conditions for the existence of solutions are examined, it will be shown that less restrictive assumptions also guarantee the existence of solutions for this new V.I. formulation of the C3F model, also ensuring that $f_{a}\left(v^{*}\right)>0, a \in \hat{E}(i), i \in \hat{N}$.

Consider the following linear programs $(21)$ defined in variables $\theta_{i}^{d}=\left(\ldots, \theta_{a}^{d}, \ldots ; a \in \hat{E}(i)\right), i \in$ $\hat{N}_{d}, d \in D$. Linear programs $(21)$ are parametrized by flows $\mathbf{v} \in \mathrm{V}$. This parametrization defines an optimal function value $w_{i}^{d}\left(\mathbf{v}_{i}^{d}\right)$ as follows:

$$
\begin{aligned}
w_{i}^{d}\left(\mathbf{v}_{i}^{d}\right)=\operatorname{Max}_{\theta_{i}^{d}} & \sum_{a \in \hat{E}(i)} v_{a}^{d} \theta_{a}^{d} \\
\text { s.t. } & \sum_{a \in \hat{E}(i)} f_{a}(v) \theta_{a}^{d}=1, \quad i \in \hat{N}_{d}, \quad d \in D \\
& \theta_{a}^{d} \geq 0
\end{aligned}
$$

Let $\Theta_{i}^{d}\left(\mathbf{v}_{i}^{d}\right)$ be the solution set of linear program $(21)$ for $d \in D, i \in \hat{N}_{d}$, and let $\Theta(\mathbf{v})=\bigotimes_{d \in D} \bigotimes_{i \in \hat{N}_{d}} \Theta_{i}^{d}\left(\mathbf{v}_{i}^{d}\right)$. Let $T^{d}\left(v, \theta^{d}\right)=\left(\ldots \psi_{a}^{d}\left(v, \theta_{a}^{d}\right) \ldots ; a \in A\right)$, where functions $\psi_{a}^{d}$ are defined as

$$
\psi_{a}^{d}\left(v, \theta_{a}^{d}\right)=\left\{\begin{array}{ll}
t_{a}(v)+\theta_{a}^{d}, & a \in \hat{E}(i) \\
t_{a}(v) & a \in E(i) \backslash \hat{E}(i)
\end{array} \quad d \in D, i \in N_{d}\right.
$$

It will be shown in this section that solution flows of the transit equilibrium model C3F satisfy the following sets of relationships:

$$
\begin{aligned}
& \mathbf{v} \in \mathrm{V} \\
& t_{a}(v)=\lambda_{i}^{d}-\lambda_{j}^{d}+\xi_{a}^{d}-\theta_{a}^{d}, \quad d \in D, \quad i \in \hat{N}_{d}, a=(i, j) \in \hat{E}(i), \\
& t_{a}(v)=\lambda_{i}^{d}-\lambda_{j}^{d}+\xi_{a}^{d}, \quad d \in D, \quad i \in N_{d}, a=(i, j) \in E(i) \backslash \hat{E}(i), \\
& v_{a}^{d} \geq 0, \quad \xi_{a}^{d} \geq 0, \quad v_{a}^{d} \xi_{a}^{d}=0,
\end{aligned}
$$

where, $\lambda_{i}^{d}$ will play the role of origin-destination travel times, as it will be shown later. The values of $\theta_{a}^{d}$ are (generally non-unique) solutions of linear programs (21), and $\xi_{a}^{d}$ values are simply dual variables of non-negativity constraints on flow variables, $v_{a}^{d} \geq 0$. By convention, it is assumed that $\lambda_{d}^{d} \triangleq 0, \forall d \in D$. It should be noticed that programs (21) are duals of the following ones:

$$
\begin{aligned}
w_{i}^{d}\left(\mathbf{v}_{i}^{d}\right)=\operatorname{Min}_{\alpha_{i}^{d}} & \alpha_{i}^{d} \\
\text { s.t. } & \frac{v_{a}^{d}}{f_{a}(v)} \leq \alpha_{i}^{d}, \quad \mid \theta_{a}^{d}, \quad a \in \hat{E}(i)
\end{aligned}, i \in \hat{N}_{d}, \quad d \in D
$$

so that optimal function values $w_{i}^{d}\left(\mathbf{v}_{i}^{d}\right)$ of linear programs (21) can be expressed also as:

$$
w_{i}^{d}\left(\mathbf{v}_{i}^{d}\right)=\operatorname{Max}_{a \in \hat{E}(i)}\left\{\frac{v_{a}^{d}}{f_{a}(v)}\right\}, i \in \hat{N}_{d}, d \in D
$$

The following complementarity conditions must hold for programs (27)

$$
\begin{aligned}
& w_{i}^{d}\left(\mathbf{v}_{i}^{d}\right) f_{a}(v)-v_{a}^{d} \geq 0, \quad \theta_{a}^{d} \geq 0, \quad d \in D, i \in \hat{N}_{d}, a \in \hat{E}(i), \\
& \left(w_{i}^{d}\left(\mathbf{v}_{i}^{d}\right) f_{a}(v)-v_{a}^{d}\right) \theta_{a}^{d}=0
\end{aligned}
$$


where variables $\theta_{a}^{d}$ verify:

$$
\sum_{a \in \hat{E}(i)} f_{a}(v) \theta_{a}^{d}=1
$$

It will be assumed that there exist solutions for the system of equations (23) through (26). In fact, this will follow automatically because it will be proved in theorem 4.5 below that solutions of the system of equations (23) through (26) are global minima of the minimization problem (18) and vice versa. Let us now consider the following sets for any feasible flow $\mathbf{v} \in \mathrm{V}$ :

$$
\begin{gathered}
\hat{E}_{*}^{d}(i, \mathbf{v})=\left\{a \in \hat{E}(i) \mid w_{i}^{d}\left(\mathbf{v}_{i}^{d}\right)=\frac{v_{a}^{d}}{f_{a}(v)}\right\}, d \in D, i \in \hat{N} \\
\hat{E}_{+}^{d}(i, \mathbf{v})=\left\{a \in \hat{E}(i) \mid v_{a}^{d}>0\right\}, d \in D, i \in \hat{N}
\end{gathered}
$$

The following two properties are readily verified:

Property 4.1 Assume that $\mathbf{v} \in \mathrm{V}$. If, at a node $i \in \hat{N}$ for $d \in D$, the total inflow at emerging links with finite frequency is positive, i.e., $\mathbf{v}_{i}^{d}=\sum_{a \in \hat{E}(i)} v_{a}^{d}>0$, then $\hat{E}_{*}^{d}(i, \mathbf{v}) \subseteq \hat{E}_{+}^{d}(i, \mathbf{v}) \subseteq \hat{E}(i)$.

Property 4.2 Assume that $\left(\mathbf{v}^{*}, \theta, \lambda, \xi\right)$ is a solution of the system of equations (23) through (26). Then, if $v^{*}=\sum_{d \in D} \mathbf{v}^{d, *}$ is the vector of total link flows, for any set $\mathcal{E}(i)$ of emerging links at node $i \in \hat{N}_{d}, d \in$ $D$, such that $\hat{E}_{*}^{d}\left(i, \mathbf{v}^{*}\right) \subseteq \mathcal{E}(i) \subseteq \hat{E}_{+}^{d}\left(i, \mathbf{v}^{*}\right)$, it must hold that

$$
\sum_{a \in \mathcal{E}(i)} f_{a}(v) \theta_{a}^{d}=1, d \in D, i \in \hat{N}_{d}
$$

Proof: Proof follows directly because of complementarity conditions (29) and (30) and dual feasibility condition (31) at $\mathbf{v}^{*}$.

The following lemma proves that solutions of the system of equations (23) through (26) are also global minima of the gap function $G_{\mathrm{CCF}}$.

Lemma 4.3 Let $\theta \in \Theta(\mathbf{v})$ be the solution set of linear programs (21), and assume that $(\mathbf{v}, \theta, \lambda)$ verify (23) through (26). Then, if $v=\sum_{d \in D} \mathbf{v}^{d}$,

1. the gap function $G_{\mathrm{CCF}}$ vanishes at $\mathbf{v}$, i.e., $G_{\mathrm{CCF}}(\mathbf{v})=0$, with $\tau_{i}^{d}(v)$ taken as $\lambda_{i}^{d}$ in $(17)$

2. the values of $(\lambda, \mathbf{v})$ verify the following generalized Bellman relationships:

$$
\lambda_{i}^{d}=\operatorname{Min}\left\{\hat{\lambda}_{i}^{d}, \check{\lambda_{i}^{d}}\right\}, \quad i \in N, d \in D
$$

with

$$
\begin{gathered}
\hat{\lambda}_{i}^{d}= \begin{cases}\operatorname{Min}_{\mathcal{E}(i) \subseteq \hat{E}(i)}\left\{\frac{1+\sum_{a \in \mathcal{E}(i)}\left(\lambda_{j_{a}}^{d}+t_{a}(v)\right) f_{a}(v)}{\sum_{a \in \mathcal{E}(i)} f_{a}(v)}\right\} \begin{array}{l}
\text { if } \hat{E}(i) \neq \emptyset \\
\text { if } \hat{E}(i)=\emptyset \\
\text { if } i=d
\end{array} \quad i \in N \\
\check{\lambda}_{i}^{d}=\left\{\begin{array}{ll}
\operatorname{Min}_{a \in E(i) \backslash \hat{E}(i)}\left\{\lambda_{j_{a}}^{d}+t_{a}(v)\right\} & \text { if } E(i) \backslash \hat{E}(i) \neq \emptyset \\
0 & \text { if } E(i) \backslash \hat{E}(i)=\emptyset \quad i \in N
\end{array} \quad \text { if } i=d\right.\end{cases}
\end{gathered}
$$

so that $\lambda_{i}^{d} \equiv \tau_{i}^{d}(v)$ as defined in (35), or equivalently in (15), and thus $\lambda_{i}^{d}$ can be interpreted as the expected travel time to destination $d \in D$ from node $i \in \hat{N}$, with the convention $\lambda_{d}^{d}=0$.

3. The gap function $G_{\mathrm{CCF}}$ decomposes in $G_{\mathrm{CCF}}(\mathbf{v})=\sum_{d \in D} G_{\mathrm{CCF}}^{d}(\mathbf{v})$, where each of the functions $G_{\mathrm{CCF}}^{d}$ vanishes at $\mathbf{v}$, i.e., $G_{\mathrm{CCF}}^{d}(\mathbf{v})=0$. 
At this point, it must be remarked that expression (35) for origin-destination travel times is equivalent to the one given in Cominetti and Correa (2001) or in Cepeda, Cominetti and Florian (2006), although it appears to be differently formulated because of the explicit treatment for infinite frequency links given in this paper.

Proof of lemma 4.3: Because of (30) and (31),

$$
w_{i}^{d}\left(\mathbf{v}_{i}^{d}\right)=\sum_{a \in \hat{E}(i)} \theta_{a}^{d} f_{a}(v) w_{i}^{d}\left(\mathbf{v}_{i}^{d}\right) \geq \sum_{a \in \hat{E}(i)} \theta_{a}^{d} v_{a}^{d}, \quad i \in \hat{N}
$$

Because of linear programs (24) and the complementarities (26),

$$
v_{a}^{d}\left(t_{a}(v)+\lambda_{j}^{d}\right)+v_{a}^{d} \theta_{a}^{d}=\lambda_{i}^{d} v_{a}^{d}, \quad d \in D, i \in \hat{N}_{d}, a=(i, j) \in \hat{E}(i)
$$

and thus, for a given destination $d \in D$, summing all the emerging links of node $i \in \hat{N}_{d}$,

$$
\begin{gathered}
w_{i}^{d}\left(\mathbf{v}_{i}^{d}\right)+\sum_{a \in E(i)} v_{a}^{d}\left(t_{a}(v)+\lambda_{j_{a}}\right)=\lambda_{i}^{d} \sum_{a \in E(i)} v_{a}^{d}=\lambda_{i}^{d}\left(g_{i}^{d}+\sum_{a \in I(i)} v_{a}^{d}\right)=\lambda_{i}^{d} g_{i}^{d}+\sum_{a \in I(i)} v_{a}^{d} \lambda_{i}^{d}, \quad d \in D, i \in \hat{N}_{d} \\
w_{i}^{d}\left(\mathbf{v}_{i}^{d}\right)+\sum_{a \in E(i)} v_{a}^{d} t_{a}(v)=\lambda_{i}^{d} g_{i}^{d}+\sum_{a \in I(i)} v_{a}^{d} \lambda_{i}^{d}-\sum_{a \in E(i)} v_{a}^{d} \lambda_{j_{a}}^{d}, \quad d \in D, i \in \hat{N}_{d}
\end{gathered}
$$

whereas for a node $i \in N_{d} \backslash \hat{N}_{d}$

$$
\sum_{a \in E(i)} v_{a}^{d} t_{a}(v)=\lambda_{i}^{d} g_{i}^{d}+\sum_{a \in I(i)} v_{a}^{d} \lambda_{i}^{d}-\sum_{a \in E(i)} v_{a}^{d} \lambda_{j_{a}}^{d}, \quad d \in D, i \in N_{d} \backslash \hat{N}_{d}
$$

but

$$
\sum_{i \in N}\left(\sum_{a \in I(i)} v_{a}^{d} \lambda_{i}^{d}-\sum_{a \in E(i)} v_{a}^{d} \lambda_{j_{a}}^{d}\right)=0, \quad d \in D
$$

where it has to be taken into account that $\lambda_{d}^{d} \triangleq 0$ and $v_{a}^{d} \triangleq 0, \forall a \in E(d)$. Then, summing from (40) and (41) for $i \in N$ and taking into account (42)

$$
G_{\mathrm{CCF}}^{d}(\mathbf{v}) \triangleq \sum_{a \in A} v_{a}^{d} t_{a}(v)+\sum_{i \in \hat{N}_{d}} w_{i}^{d}\left(\mathbf{v}_{i}^{d}\right)-\sum_{i \in N_{d}} \lambda_{i}^{d} g_{i}^{d}=0 \Rightarrow G_{\mathrm{CCF}}(\mathbf{v})=0
$$

where, because of $(21), w_{i}^{d}\left(\mathbf{v}_{i}^{d}\right)=\underset{a \in \hat{E}(i)}{\operatorname{Max}}\left\{\frac{v_{a}^{d}}{f_{a}(v)}\right\}$, and assertions 1 and 3 of the lemma have been proved.

Now, the equivalence between variables $\lambda_{i}^{d}$ and origin-destination travel times $\tau_{i}^{d}(v)$ will be proved. Assume $v_{i}^{d}>0$ and let $d \in D$ and $i \in N_{d}$. Then, because of the complementarity conditions (26)

$$
\begin{gathered}
\left(t_{a}(v)+\lambda_{j}^{d}\right) f_{a}(v)+\theta_{a}^{d} f_{a}(v) \geq \lambda_{i}^{d} f_{a}(v), a=(i, j) \in \hat{E}(i), i \in \hat{N}_{d}, \quad d \in D, \\
t_{a}(v)+\lambda_{j}^{d} \geq \lambda_{i}^{d}, \quad a=(i, j) \in E(i) \backslash \hat{E}(i), i \in N_{d}, \quad d \in D
\end{gathered}
$$

where (44) is verified with equality if $a=(i, j) \in \hat{E}_{+}^{d}(i, \mathbf{v}) \subseteq \hat{E}(i)$. Now, let $\mathcal{E}(i) \subseteq \hat{E}(i)$ be a subset of links within a strategy at node $i \in \hat{N}_{d}$. Then,

$$
\lambda_{i}^{d} \sum_{a \in \mathcal{E}(i)} f_{a}(v) \stackrel{(1)}{\leq} \sum_{a \in \mathcal{E}(i)}\left(t_{a}(v)+\lambda_{j_{a}}^{d}\right) f_{a}(v)+\sum_{a \in \mathcal{E}(i)} \theta_{a}^{d} f_{a}(v) \stackrel{(2)}{\leq} 1+\sum_{a \in \mathcal{E}(i)}\left(t_{a}(v)+\lambda_{j_{a}}^{d}\right) f_{a}(v)
$$

Now, let $\hat{\lambda}_{i}^{d}$ and $\check{\lambda}_{i}^{d}$ be defined for a node $i \in N$ and destination $d \in D$, as in expressions (36) and (37). Because $v_{i}^{d}>0$, some of the above inequalities (44), (45) must be verified as equalities, and because of property 4.2 , inequalities (1) and (2) in (46) must be verified as equalities for some $\mathcal{E}(i)$, if it is the case that $i \in \hat{N}_{d}$. Then, 


$$
\lambda_{i}^{d}=\operatorname{Min}\left\{\hat{\lambda_{i}^{d}}, \check{\lambda_{i}^{d}}\right\}, \quad i \in N, \quad d \in D
$$

whereas simply $\lambda_{i}^{d} \leq \operatorname{Min}\left\{\hat{\lambda_{i}^{d}}, \check{\lambda_{i}^{d}}\right\}$ if $v_{i}^{d}=0$

Thus, a solution $(\mathbf{v}, \theta, \lambda)$ of (23) through (26) is such that $\mathbf{v} \in \mathrm{V}^{*}$ and $\lambda_{i}^{d}$ are the origin-destination expected travel times from nodes $i \in N$ to destinations $d \in D$. The following lemma proves that the converse is also true.

Lemma 4.4 Let $\mathbf{v}^{*} \in \mathrm{V}^{*}$; then, $\exists \theta_{a}^{d}, \lambda_{i}^{d} \equiv \tau_{i}^{d}\left(\mathbf{v}^{*}\right), \xi_{a}^{d} \geq 0$ so that relationships (23) through (26) are verified for $\mathbf{v}^{*}$, and $\theta_{a}^{d}$ are, precisely, solutions of linear programs (21).

Proof: Because $\mathbf{v}^{*} \in \mathrm{V}^{*}$, theorem 3.3 in Cepeda et al. (2006) states that for $i \in N_{d}, \exists \alpha_{i}^{d} \geq 0$ that are solutions of linear programs (27), i.e.: $\alpha_{i}^{d} \equiv w_{i}^{d}\left(\mathbf{v}_{i}^{d}\right)$. On the other hand, solutions of linear programs (21) and (27) will verify complementary slack conditions (29), (30) and dual feasibility (31).

Now, let $\beta_{a}^{d}$, arbitrary constants $r_{i}^{d}>0$ and $\eta_{a}^{d} \triangleq t_{a}(v)+\tau_{j_{a}}^{d}(v)-\tau_{i}^{d}(v)+\beta_{a}^{d} r_{i}^{d}$ so that for $\alpha_{i}^{d} \geq 0$ the following relationship is verified:

$$
\begin{gathered}
t_{a}(v)+\tau_{j_{a}}^{d}(v)-\tau_{i}^{d}(v)=-\beta_{a}^{d} r_{i}^{d}+\eta_{a}^{d} \\
\text { Case a) } \Rightarrow \frac{v_{a}^{d}}{f_{a}(v)}=\alpha_{i}^{d} \text { then }, \quad \beta_{a}^{d}>0, \quad \eta_{a}^{d}=0, a \in \hat{E}^{*}(i) \\
\text { Case b) } \Rightarrow\left\{\begin{array}{l}
\frac{v_{a}^{d}}{f_{a}(v)}=\alpha_{i}^{d} \text { then } \quad \beta_{a}^{d}=0, \eta_{a}^{d}=0, a \in \hat{E}^{*}(i) \\
0<\frac{v_{a}^{d}}{f_{a}(v)}<\alpha_{i}^{d} \text { then } \quad \beta_{a}^{d}=0, \eta_{a}^{d}=0, a \in \hat{E}^{+}(i)
\end{array}\right. \\
\text { Case c) } \quad \Rightarrow \frac{v_{a}^{d}}{f_{a}(v)}=0 \text { then }, \quad \beta_{a}^{d}=0, \eta_{a}^{d}>0, a \in \hat{E}(i) \backslash \hat{E}^{+}(i)
\end{gathered}
$$

Relationships (49), (50) and (51) show that, as $0<f_{a}(v)<+\infty$,

$$
\left(\alpha_{i}^{d} f_{a}(v)-v_{a}^{d}\right) \beta_{a}^{d} r_{i}^{d}=0, \quad \alpha_{i}^{d} f_{a}(v)-v_{a}^{d} \geq 0, \beta_{a}^{d} \geq 0
$$

and $r_{i}^{d}>0$ can be chosen so that:

$$
r_{i}^{d} \sum_{a \in \hat{E}(i)} f_{a}(v) \beta_{a}^{d}=1
$$

Additionally, from (49), (50) and (51):

$$
\eta_{a}^{d} v_{a}^{d}=0, \quad \eta_{a}^{d} \geq 0, v_{a}^{d} \geq 0
$$

Thus, it has been proved that relationships (24) through (26), are verified with $\theta_{a}^{d}=r_{i}^{d} \beta_{a}^{d}, \xi_{a}^{d}=$ $\eta_{a}^{d}, a \in \hat{E}(i)$ and $\lambda_{i}^{d}=\tau_{i}^{d}(v), i \in \hat{N}$ and that $r_{i}^{d} \beta_{a}^{d}$ solve linear programs (21).

From previous lemmas 4.3 and 4.4 , the following theorem is derived:

Theorem 4.5 The following two statements are equivalent:

1. $\mathbf{v} \in \mathrm{V}^{*}$

2. The vector of flows $\mathbf{v} \in \mathrm{V}$, is a solution of (24) through (26), and $\theta_{a}^{d}, d \in D, a \in \hat{E}(i), i \in \hat{N}$ are solutions of linear programs (21)

and $\lambda_{i}^{d}$ are the expected origin-destination travel times from node $i \in N$ to destination $d \in D$, defined recursively by $(15)$, i.e., $\lambda_{i}^{d}=\tau_{i}^{d}(v)$. 


\subsection{Reformulation as a VI}

Let us consider the convex and non-differentiable function $H_{n}(\cdot): \mathbb{R}^{n} \rightarrow \mathbb{R}, H_{n}(x)=\max { }_{1 \leq \ell \leq n}\left\{x_{\ell}\right\}$. Let $\mathcal{I}(x)=\left\{1 \leq \ell \leq n \mid x_{\ell}=H_{n}(x)\right\}$. Then the convex subdifferential, $\partial H_{n}(x)$, of function $H_{n}(\cdot)$ at a point $x \in \mathbb{R}_{+}^{n}$ is the set:

$$
\partial H_{n}(x)=\left\{\alpha \in \mathbb{R}_{+}^{n} \mid \sum_{\ell \in \mathcal{I}(x)} \alpha_{\ell}=1, \quad \alpha_{\ell}=0 \text { if } \ell \notin \mathcal{I}(x)\right\}, x \in \mathbb{R}_{+}^{n}
$$

Now, let $x(\mathbf{v})=\left(\ldots, x_{i}^{d}(\mathbf{v}), \ldots ; i \in \hat{N}, d \in D\right)$ with $x_{i}^{d}(\mathbf{v})=\left(\ldots, x_{a}^{d}(\mathbf{v}), \ldots ; a \in \hat{E}(i)\right), d \in D, i \in \hat{N}_{d}$ and $x_{a}^{d}(\mathbf{v})=v_{a}^{d} \sigma_{a}(v)$. Functions $w_{i}^{d}\left(\mathbf{v}_{i}^{d}\right)$ can be rewritten as

$$
w_{i}^{d}\left(\mathbf{v}_{i}^{d}\right)=H_{|\hat{E}(i)|}\left(x_{i}^{d}(\mathbf{v})\right), \quad d \in D, i \in \hat{N}_{d}
$$

Recall that because of assumption 3.2, it can be conjectured that $f_{a}(v)>0$ at the solutions of (23) through $(26)$ and $\sigma_{a}(v)=1 / f_{a}(v)<+\infty, a \in \hat{E}(i), i \in \hat{N}$. Now, new variables $\zeta_{a}^{d}$ can be introduced, whose relationship with $\theta_{a}^{d}$ 's is simply by means of $\theta_{a}^{d}=\sigma_{a}(v) \zeta_{a}^{d}$. Linear programs (21) can be now restated as:

$$
\begin{array}{cl}
w_{i}^{d}\left(\mathbf{v}_{i}^{d}\right)=\operatorname{Max}_{\zeta_{i}^{d}} & \sum_{a \in \hat{E}(i)} v_{a}^{d} \sigma_{a}(v) \zeta_{a}^{d} \\
\text { s.t. } & \sum_{a \in \hat{E}(i)} \zeta_{a}^{d}=1 \quad, \quad d \in D, i \in \hat{N}_{d} \\
& \zeta_{a}^{d} \geq 0
\end{array}
$$

where $\zeta_{i}^{d}=\left(\ldots, \zeta_{a}^{d}, \ldots ; a \in \hat{E}(i)\right), i \in \hat{N}_{d}, d \in D$. Let $\mathcal{Z}_{i}^{d}\left(\mathbf{v}_{i}^{d}\right), d \in D, i \in \hat{N}_{d}$ be the solution sets of programs (57). Now, define $\mathrm{T}^{d}\left(v, \zeta^{d}\right)$ as

$$
\mathrm{T}^{d}\left(v, \zeta^{d}\right)=\left(\ldots, \Psi_{a}^{d}\left(v, \zeta_{a}^{d}\right), \ldots ; a \in A\right)
$$

where $\Psi_{a}^{d}$ are defined as:

$$
\Psi_{a}^{d}\left(v, \zeta_{a}^{d}\right)=\left\{\begin{array}{ll}
t_{a}(v)+\sigma_{a}(v) \zeta_{a}^{d}, & a \in \hat{E}(i) \\
t_{a}(v) & a \in E(i) \backslash \hat{E}(i)
\end{array} \quad d \in D, i \in N_{d}\right.
$$

With these new variables $\zeta$ and taking into account that $\mathcal{Z}_{i}^{d}\left(\mathbf{v}_{i}^{d}\right)=\partial H_{|\hat{E}(i)|}\left(x_{i}^{d}(\mathbf{v})\right)$, system of equations (23) through (26) can be rewritten as:

$$
\begin{aligned}
& \mathbf{v} \in \mathrm{V} \\
& t_{a}(v)+\zeta_{a}^{d} \sigma_{a}(v)=\lambda_{i}^{d}-\lambda_{j}^{d}+\xi_{a}^{d}, d \in D, \quad i \in \hat{N}_{d}, a=(i, j) \in \hat{E}(i), \\
& t_{a}(v)=\lambda_{i}^{d}-\lambda_{j}^{d}+\xi_{a}^{d}, \quad d \in D, \quad i \in N_{d}, a=(i, j) \in E(i) \backslash \hat{E}(i), \\
& v_{a}^{d} \geq 0, \quad \xi_{a}^{d} \geq 0, \quad v_{a}^{d} \xi_{a}^{d}=0,
\end{aligned}
$$

where $\zeta_{i}^{d}$ are solutions of (57). Because $\lambda_{i}^{d}$ are origin-destination travel times (as shown in lemmas 4.3 and 4.4 ), system of equations (60) through (63) can be interpreted as a set of coupled variational inequalities parametrized by $\zeta$ :

$$
\begin{aligned}
& \text { Find } \mathbf{v}^{d} \in \mathrm{V}^{d} \text { so that, } \forall \mathbf{u}^{d}, \in \mathrm{V}^{d} \text { : } \\
& \qquad \sum_{a \in A} \Psi_{a}^{d}\left(v, \zeta^{d}\right)\left(u_{a}^{d}-v_{a}^{d}\right) \geq 0, \quad d \in D
\end{aligned}
$$

where, as usual, $v=\sum_{d \in D} \mathbf{v}^{d}$. Solutions $\zeta_{i}^{d}$ of system (57) will also verify the following variational inequalities parametrized by flows $\mathbf{v}$ :

$$
\begin{aligned}
& \text { Find } \zeta_{i}^{d} \in S_{i}^{d} \text { so that, } \forall \mathbf{z}_{i}^{d} \in S_{i}^{d} \text { : } \\
& \qquad \sum_{a \in \hat{E}(i)} x_{a}^{d}(\mathbf{v})\left(z_{a}^{d}-\zeta_{a}^{d}\right) \leq 0, \quad i \in \hat{N}_{d}, d \in D
\end{aligned}
$$


Now, let $S=\bigotimes_{d \in D} \bigotimes_{i \in \hat{N}_{d}} S_{i}^{d}$. Because $H_{|\hat{E}(i)|}(\cdot)$ is the support function of the simplex set $S_{i}^{d}=$ $\left\{\alpha \in \mathbb{R}_{+}^{|\hat{E}(i)|} \mid \sum_{a \in \hat{E}(i)} \alpha_{a}=1\right\}$ associated with node $i \in \hat{N}_{d}$ and taking into account previous VIs (64) and (65), the following VI is readily derived:

$$
\left[\begin{array}{l}
\text { Find }(\mathbf{v}, \zeta) \in \mathrm{V} \times S \text { so that, } \forall(\mathbf{u}, \mathrm{z}) \in \mathrm{V} \times S: \\
\sum_{d \in D} \sum_{i \in N_{d}}\left(\sum_{a \in E(i)}\left(t_{a}(v)+s_{a}(v) \zeta_{a}^{d}\right)\left(u_{a}^{d}-v_{a}^{d}\right)-y_{a}^{d}(\mathbf{v})\left(z_{a}^{d}-\zeta_{a}^{d}\right)\right) \geq 0
\end{array}\right]
$$

where

$$
y_{a}^{d}(\mathbf{v})=\left\{\begin{array}{ll}
x_{a}^{d}(\mathbf{v})=\sigma_{a}(v) v_{a}^{d} & \text { if } a \in \hat{E}(i) \\
0 & \text { if } a \in E(i) \backslash \hat{E}(i)
\end{array} \quad d \in D, a \in E(i), i \in N_{d}\right.
$$

and

$$
s_{a}(v)=\left\{\begin{array}{ll}
\sigma_{a}(v) & \text { if } a \in \hat{E}(i) \\
0 & \text { if } a \in E(i) \backslash \hat{E}(i)
\end{array} \quad a \in E(i), i \in N\right.
$$

The following is a restatement of previous theorem 4.5.

Theorem 4.6 The following two statements are equivalent:

$$
\begin{aligned}
& \text { 1. } \mathbf{v} \in \mathrm{V}^{*} \\
& \text { 2. }(\mathbf{v}, \zeta) \in \mathrm{V} \times S \text { is a solution of previous VI (VI) in (66) }
\end{aligned}
$$

and $\lambda_{i}^{d}$, the dual variables defined by the system of equations (60) through (63), are the expected origindestination travel times from node $i \in N$ to destination $d \in D, \tau_{i}^{d}(v)$, defined recursively by (35), (36), (37) with $\lambda_{i}^{d}=\tau_{i}^{d}(v)$.

Remark 4.7 When frequencies $f_{a}(\cdot)=r_{a}, a \in \hat{E}(i), i \in \hat{N}$ are flow independent and constant and travel costs $t_{a}(v)$ at links $a \in A$ have a diagonal and positive semi-definite jacobian, or equivalently, $t_{a}(v)=t_{a}\left(v_{a}\right)$, it can be shown that models developed in Spiess (1984) and in Spiess and Florian (1989) are reproduced. If frequencies are constant, then $x_{i}^{d}\left(\mathbf{v}_{i}^{d}\right)=\left(\ldots, v_{a}^{d} / r_{a}, \ldots ; a \in \hat{E}(i)\right)$. Now, because of $(28)$ and (56):

$$
\partial w_{i}^{d}\left(\mathbf{v}_{i}^{d}\right)=\left\{\left(\ldots, \zeta_{a}^{d} / r_{a}, \ldots ; a \in \hat{E}(i)\right) \in \mathbb{R}_{+}^{|\hat{E}(i)|} \mid \sum_{a \in \hat{E}_{*}^{d}(i, \mathbf{v})} \zeta_{a}^{d}=1, \zeta_{a}^{d}=0, a \notin \hat{E}_{*}^{d}(i, \mathbf{v})\right\}
$$

This implies that VI (VI) in (66) can be simply stated as:

$$
\begin{aligned}
& \mathbf{v} \in \mathrm{V} \\
& t_{a}\left(v_{a}\right)+\left(\partial w_{i}^{d}\left(\mathbf{v}_{i}^{d}\right)\right)_{a}=\lambda_{i}^{d}-\lambda_{j}^{d}+\xi_{a}^{d}, \quad d \in D, i \in \hat{N}_{d}, a=(i, j) \in \hat{E}(i), \\
& t_{a}\left(v_{a}\right)=\lambda_{i}^{d}-\lambda_{j}^{d}+\xi_{a}^{d}, \quad d \in D, i \in \hat{N}_{d}, a=(i, j) \in E(i) \backslash \hat{E}(i), \\
& v_{a}^{d} \geq 0, \quad \xi_{a}^{d} \geq 0, \quad v_{a}^{d} \xi_{a}^{d}=0,
\end{aligned}
$$

which are, in fact, first-order conditions of the following optimization problem for the semicongested transit assignment problem in Spiess and Florian (1989):

$$
\begin{array}{ll}
\operatorname{Min}_{\mathbf{v}, w} & \sum_{a \in A} \int_{0}^{v_{a}} t_{a}(\alpha) d \alpha+\sum_{d \in D} \sum_{i \in \hat{N}_{d}} w_{i}^{d} \\
\text { s.t: } & v_{a}=\sum_{d \in D} v_{a}^{d}, a \in A \\
& v_{a}^{d} \leq r_{a} w_{i}^{d}, a \in \hat{E}(i), i \in \hat{N}, d \in D \\
& \mathbf{v} \in \mathrm{V}
\end{array}
$$


Additionally, (70) to (73) are optimality conditions for the transit assignment model in Spiess (1984) for $t_{a}\left(v_{a}\right)=t_{a}=$ ctant, which will be designated by $[\mathrm{PL}](r, t)$ :

$$
\begin{aligned}
\operatorname{Min}_{\mathbf{v}, w} & \sum_{d \in D} \sum_{a \in A} t_{a} v_{a}^{d}+\sum_{d \in D} \sum_{i \in \hat{N}_{d}} w_{i}^{d} \\
\text { [PL] }(r, t) \quad \text { s.t }: \quad & v_{a}^{d} \leq r_{a} w_{i}^{d}, a \in \hat{E}(i), i \in \hat{N}, d \in D \\
& \mathbf{v} \in \mathrm{V}
\end{aligned}
$$

\subsection{Relationship of $G_{\mathrm{CCF}}$ with the primal gap function}

The functional $F(\cdot, \cdot): \mathrm{V} \times S \rightarrow \mathbb{R}^{m}\left(m=|D| \cdot|A|+\sum_{d \in D} \sum_{i \in \hat{N}_{d}}|\hat{E}(i)|\right)$ for variational inequality (VI) in (66) is:

$$
F(\mathbf{v}, \zeta)=\left(\begin{array}{ll}
\mathrm{T}^{d}\left(v, \zeta^{d}\right) & ; d \in D \\
-\sigma_{a}(v) v_{a}^{d} & ; a \in \hat{E}(i), i \in \hat{N}_{d}, d \in D
\end{array}\right)
$$

or more explicitly:

$$
F(\mathbf{v}, \zeta)=\left(\frac{F_{v}(\mathbf{v}, \zeta)}{F_{\zeta}(\mathbf{v})}\right)=\left(\begin{array}{cl}
t_{a}(v)+\zeta_{a}^{d} \sigma_{a}(v) & ; a \in \hat{E}(i), i \in \hat{N}_{d}, d \in D \\
t_{a}(v) & ; a \in E(i) \backslash \hat{E}(i), i \in N_{d}, d \in D \\
\hline-\sigma_{a}(v) v_{a}^{d} & ; a \in \hat{E}(i), i \in \hat{N}_{d}, d \in D
\end{array}\right)
$$

If now $Q=\mathrm{V} \times S, x=(\mathbf{v}, \zeta), y=(\mathbf{u}, z)$, then (VI) in (66) will be rewritten simply as: Find $x \in Q$ so that $F(x)^{\top}(y-x) \geq 0, \forall y \in Q$, and its primal gap function $G_{P}(x)$ will be:

$$
G_{P}(x)=\operatorname{Max}_{y \in Q} F(x)^{\top}(x-y)
$$

Let us, for simplicity, denote $x=(\mathbf{v}, \zeta)$ and $y=(\mathbf{u}, z)$ for $x, y \in \mathrm{V} \times S$. Then, it must be noted that if $F(\cdot)$ is the functional defined in $(77)$,

$$
\begin{gathered}
x^{\top} F(x)=\sum_{a \in A} v_{a} t_{a}(v) \\
y^{\top} F(x)=\sum_{a \in A} u_{a} t_{a}(v)+\sum_{d \in D} \sum_{i \in \hat{N}_{d}} \sum_{a \in \hat{E}(i)} \sigma_{a}(v)\left(\zeta_{a}^{d} u_{a}^{d}-z_{a}^{d} v_{a}^{d}\right)
\end{gathered}
$$

The following theorem 4.9 states the relationship between the gap $G_{\mathrm{CCF}}$ in Cepeda et al. (2006) and the primal gap function $G_{P}$ for (VI) in (66). For convenience, the gap function $G_{\mathrm{CCF}}(\mathbf{v})$ in $(17)$ will expressed as the difference of two functions, namely:

$$
G_{\mathrm{CCF}}(\mathbf{v})=G_{\mathrm{CCF}}^{(0}(\mathbf{v})-G_{\mathrm{CCF}}^{(1}(v)
$$

where $G_{\mathrm{CCF}}^{(0}(\mathbf{v})$ and $G_{\mathrm{CCF}}^{(1}(v)$ are defined as:

$$
\begin{gathered}
G_{\mathrm{CCF}}^{(0}(\mathbf{v}) \triangleq \sum_{d \in D}\left[\sum_{a \in A} v_{a}^{d} t_{a}(v)+\sum_{i \in \hat{N}_{d}} \operatorname{Max}_{a \in \hat{E}(i)}\left\{\frac{v_{a}^{d}}{f_{a}(v)}\right\}\right] \\
G_{\mathrm{CCF}}^{(1}(v) \triangleq \sum_{d \in D} \sum_{i \in N_{d}} g_{i}^{d} \tau_{i}^{d}(v)
\end{gathered}
$$

and $\tau_{i}^{d}(v)$ are the travel times from node $i$ to destination $d$ defined in (15). Function $G_{\text {CCF }}^{(1}(v)$ is then the total travel time spent on the network with constant travel costs at links fixed at $t(v)$ and constant frequencies fixed at $f(v)$.

Lemma 4.8 Function $G_{\mathrm{CCF}}^{(1}(v)$ can be evaluated as the optimal objective value of the following program:

$$
G_{\mathrm{CCF}}^{(1}(v)=\operatorname{Min} \mathbf{u} \in \mathrm{V} \sum_{d \in D}\left[\sum_{a \in A} t_{a}(v) u_{a}^{d}+\sum_{i \in \hat{N}_{d}} \operatorname{Max}_{a \in \hat{E}(i)}\left\{\frac{u_{a}^{d}}{f_{a}(v)}\right\}\right]
$$


Proof: The optimization problem in the right-hand side of (84) is, in fact, the linear problem [PL] $(f(v), t(v))$. Because of duality in linear programming, its optimal objective function value is $\sum_{d \in D} \sum_{i \in N_{d}} g_{i}^{d} \lambda_{i}^{d}$, being $\lambda_{i}^{d}$ the travel time from node $i$ to destination $d$. Because $\lambda_{i}^{d}=\tau_{i}^{d}(v)$, there follows (84).

As a consequence of previous lemma 4.8 , another convenient way of expressing gap function $G_{\mathrm{CCF}}$ will be:

$$
G_{\mathrm{CCF}}(\mathbf{v})=\varphi(\mathbf{v}, v)-\operatorname{Min} \mathbf{u} \in \mathrm{V} \varphi(\mathbf{u}, v)
$$

where function $\varphi(\cdot, \cdot)$ is defined as:

$$
\varphi(\mathbf{u}, v) \triangleq \sum_{d \in D}\left[\sum_{a \in A} t_{a}(v) u_{a}^{d}+\sum_{i \in \hat{N}_{d}} \operatorname{Max}_{a \in \hat{E}(i)}\left\{\frac{u_{a}^{d}}{f_{a}(v)}\right\}\right]
$$

and obviously, $G_{\mathrm{CCF}}^{(0}(\mathbf{v})=\varphi(\mathbf{v}, v), G_{\mathrm{CCF}}^{(1}(v)=\operatorname{Min} \mathbf{u} \in \mathrm{V} \varphi(\mathbf{u}, v)$.

Theorem 4.9 Let $\mathbf{v} \in \mathrm{V}$ be a feasible flow. Then,

$$
G_{\mathrm{CCF}}(\mathbf{v})=\operatorname{Min}_{\zeta \in S} G_{P}(\mathbf{v}, \zeta)
$$

Proof:

$$
\begin{aligned}
& \operatorname{Min}_{\zeta \in S} G_{P}(\mathbf{v}, \zeta)= \\
& =\operatorname{Min}_{\zeta \in S} \operatorname{Max}_{\substack{\mathbf{u} \in \mathrm{V} \in S \\
\text { int }}}\left[\sum_{a \in A} t_{a}(v) v_{a}^{d}+\sum_{i \in \hat{N}_{d}} \sum_{a \in \hat{E}(i)}\left(z_{a}^{d} \sigma_{a}(v) v_{a}^{d}-\zeta_{a}^{d} \sigma_{a}(v) u_{a}^{d}\right)-\sum_{a \in A} t_{a}(v) u_{a}^{d}\right] \stackrel{(1)}{=} \\
& =\sum_{d \in D}\left[\sum_{a \in A} t_{a}(v) v_{a}^{d}+\sum_{i \in \hat{N}_{d}} \operatorname{Max}_{a \in \hat{E}(i)}\left\{\sigma_{a}(v) v_{a}^{d}\right\}\right]- \\
& \quad-\operatorname{Max}_{\zeta \in S} \operatorname{Min} \mathbf{u} \in \mathrm{V} \sum_{d \in D}\left[\sum_{a \in A} t_{a}(v) u_{a}^{d}+\sum_{i \in \hat{N}_{d}} \sum_{a \in \hat{E}(i)} \zeta_{a}^{d} \sigma_{a}(v) u_{a}^{d}\right] \stackrel{(2)}{=} \\
& =\sum_{d \in D}\left[\sum_{a \in A} t_{a}(v) v_{a}^{d}+\sum_{i \in \hat{N}_{d}} \operatorname{Max}_{a \in \hat{E}(i)}\left\{\sigma_{a}(v) v_{a}^{d}\right\}\right]- \\
& \quad-\operatorname{Min}_{\mathbf{u} \in \mathrm{V}} \sum_{d \in D}\left[\sum_{a \in A} t_{a}(v) u_{a}^{d}+\sum_{i \in \hat{N}_{d}} \operatorname{Max}_{a \in \hat{E}(i)}\left\{\sigma_{a}(v) u_{a}^{d}\right\}\right] \stackrel{(3)}{=} G_{\mathrm{CCF}}^{(0}(\mathbf{v})-G_{\mathrm{CCF}}^{(1)}(v)
\end{aligned}
$$

Equality (1) follows after maximizing in $z$ terms $\sum_{a \in \hat{E}(i)} \sigma_{a}(v) v_{a}^{d} z_{a}^{d}$ on simplex set $S_{i}^{d}$, i.e., linear programs (57) with optimization variables $z_{a}^{d}$ instead of $\zeta_{a}^{d}$. Equality (2) follows from the fact that $\operatorname{Min}_{\mathbf{u} \in V} \operatorname{Max}_{\zeta \in S}$ can be interchanged with $\operatorname{Max}_{\zeta \in S} \operatorname{Min}_{\mathbf{u} \in V}$ because products $\zeta_{a}^{d} u_{a}^{d}$ are concave-convex and set $S$ is bounded (see, for instance, corollary 37.3.2, page 393, in Rockafellar (1972)). Again, linear problems (57) appear. The last equality (3) follows directly from previous lemma 4.8.

Proposition 4.10 ( Characterization of C3F equilibrium as a fixed-point inclusion ). Let [PL] $(r, t)$ ) be the linear program defined in (75) for the uncongested transit assignment model with constant frequencies $r$ and travel times $t$. Then, $\mathbf{v}^{*} \in \mathrm{V}^{*}$ iff:

$$
\mathbf{v}^{*} \in \operatorname{Sol}\left([\mathrm{PL}]\left(f\left(v^{*}\right), t\left(v^{*}\right)\right)\right)=\operatorname{Sol} \operatorname{Min} \mathbf{u} \in \mathrm{V} \varphi\left(\mathbf{u}, v^{*}\right)
$$

Proof: Proof is immediate because of lemma 4.8 and the definition of gap function $G_{\mathrm{CCF}}$, which vanishes at equilibrium points of the $\mathrm{C} 3 \mathrm{~F}$ model.

Remark 4.11 Notice that, in view of proposition 4.10, the MSA heuristic procedure to solve the C3F model used in Cepeda et al. (2006) can be interpreted as the calculation of a solution for the fixed-point point inclusion (89). 


\section{Existence of solutions and capacitated models}

Because it has been proved in previous sections that the C3F model can be formulated as a VI, in this section, the question of the existence of solutions is explored from this perspective using general and wellknown results from the classical theory of variational inequalities. Assumptions required for the existence of solutions are, under this scope, much less stringent than those stated in Cominetti and Correa (2001). The existence of solutions for models with additional constraints on which travel time and efff functions become $+\infty$ on the boundaries imposed by these constraints is also analyzed in a very general setting.

Specifically for this section the following notations will be used:

If $M$ is a convex polyhedron, let vertex $(M)$ and $\operatorname{ray}(M)$ denote its set of vertexes and rays, respectively, and $\hat{M}=\operatorname{Hull}(\operatorname{vertex}(M))$ and $\vec{M}=\operatorname{Pos}(\operatorname{ray}(M))$ denote the convex hull of all vertexes and the cone of all rays, respectively, in the set $M$.

By $B_{a}, \mathbf{B}_{a}, \mathbb{B}_{a}$ it will be denoted balls with radius $a$ on $\mathbb{R}^{n}$ centered at 0 , for $n=|A|,|D||A|,|D||A|+$ $\sum_{d \in D} \sum_{i \in \hat{N}_{d}}|\hat{E}(i)|$, respectively.

For a set $M \subset \mathbb{R}^{n}$, its topological interior will be designated by int $M$, and its frontier will be designated by $\operatorname{fr}(M)$, i.e., $\operatorname{fr}(M)=\left\{y \notin M \mid \forall \epsilon>0, B_{\epsilon}(y) \cap M \neq \emptyset\right\}$. The Euclidean distance from a point $y \in \mathbb{R}^{n}$ to $M$ is denoted by $\operatorname{dist}(y, M)$.

Finally, recall that for a VI, find $x \in M$ so that $\Phi(x)^{\top}\left(x^{\prime}-x\right) \geq 0, \forall x^{\prime} \in M$, it is known that if solutions $x^{*}$ exist, then they verify the following inclusion (see, for example Goh and Yang (2002), page 194):

$$
x^{*} \in \text { Sol Min } x \in M x^{\top} \Phi\left(x^{*}\right)
$$

Taking into account relationship (80), inclusion (90) for an arbitrary V.I. can be expressed for $\mathrm{VI}(F, \mathrm{~V})$ in (66) as:

$$
\left(\begin{array}{c}
\mathbf{u} \\
z
\end{array}\right) \in \operatorname{Sol}\left(\begin{array}{c}
\operatorname{Min}_{\mathbf{v} \in \mathrm{V}} \mathbf{v}^{\top} F_{v}(\mathbf{u}, z) \\
\operatorname{Min}_{\zeta \in S} \zeta^{\top} F_{\zeta}(\mathbf{u})
\end{array}\right)
$$

The first linear program in (91) can be expressed as:

$$
\operatorname{Min}_{\mathbf{v} \in \mathrm{V}} \sum_{d \in D}\left[\sum_{a \in A} t_{a}(u) v_{a}^{d}+\sum_{i \in \hat{N}_{d}} \sum_{a \in \hat{E}(i)} z_{a}^{d} \sigma_{a}(u) v_{a}^{d}\right]
$$

whereas the second one decomposes into:

$$
\begin{array}{cl}
\operatorname{Max}_{\zeta^{d}} & \sum_{a \in \hat{E}(i)} u_{a}^{d} \sigma_{a}(u) \zeta_{a}^{d} \\
\text { s.t. } & \sum_{a \in \hat{E}(i)} \zeta_{a}^{d}=1 \quad, \quad d \in D, i \in \hat{N}_{d} \\
& \zeta_{a}^{d} \geq 0
\end{array}
$$

\subsection{Models without explicit capacity bounds}

In this subsection, congested models without sharp capacity bounds will be considered. These are models with efff's that decrease with increasing flows but do not vanish and with travel times that may increase but remain finite at any point in the feasible set of flows $\mathrm{V}$.

Theorem 5.1 Let $t_{a}(\cdot), a \in A$ and $\sigma_{a}(\cdot), a \in \hat{A}$ be continuous and positive on $\mathrm{V}$. Then, (VI) in (66) has a nonempty set of solutions, and any solution $\mathbf{v}^{*} \in \hat{\mathrm{V}}$, i.e., it contains no cyclic flows on the expanded graph.

Proof: Note that V may be an unbounded set because of possible cycles on the expanded transit network. Consider now VI in (66) but defined on $\mathrm{V}^{\prime}=\mathrm{V} \cap C$, where $C=\bigotimes_{d \in D} C_{d}$ and $C_{d}$ are boxes defined as $C_{d}=\left\{\mathbf{v}^{d} \in \mathbb{R}^{|A|} \mid 0 \leq v_{a}^{d} \leq \rho_{d}, a \in A\right\}$. Bounds $\rho_{d}$ are taken as $\rho_{d}>\sum_{(p, d) \in W} g_{p, d}$. Because functional (77) is continuous on $\mathrm{V}^{\prime}=\mathrm{V} \cap C$, which is a convex and compact set, this newly defined $\operatorname{VI}\left(F, \mathrm{~V}^{\prime}\right)$ has at least a solution $\left(\mathbf{v}^{*}, \zeta^{*}\right)$. Now, let $\rho=|A|^{1 / 2}\left(\sum_{d \in D} \rho_{d}^{2}\right)^{1 / 2}$. To see that $\left\|\mathbf{v}^{*}\right\|_{2}<\rho$, simply notice that $\mathbf{v}^{*}$ must also be a solution of the linear problem

$$
\operatorname{Min} \mathbf{u} \in \mathrm{V} \cap C \sum_{d \in D}\left[\sum_{a \in A} t_{a}\left(v^{*}\right) u_{a}^{d}+\sum_{i \in \hat{N}_{d}} \sum_{a \in \hat{E}(i)} \zeta_{a}^{d *} \sigma_{a}\left(v^{*}\right) u_{a}^{d}\right]
$$


Because $t_{a}(\cdot), \sigma_{a}(\cdot)>0$ and $\zeta_{a}^{d *} \geq 0$, cycles cannot appear on the solution of linear program (94), which may be solved by decomposing it by destinations, and thus, $\mathbf{v}^{*} \in \hat{\mathrm{V}}$. Solution flows per destination $\mathbf{v}^{d *}$ of (94) verify $\max _{a \in A}\left\{v_{a}^{d *}\right\}<\rho_{d}$ and $\left\|\mathbf{v}^{d *}\right\|_{2}<|A|^{1 / 2} \rho_{d}$, and therefore $\left\|\mathbf{v}^{*}\right\|_{2}<\rho$. Because $\otimes_{d \in D} B_{\rho_{d}|A|^{1 / 2}} \subset C \subset \mathbf{B}_{\rho}$, and $\mathbf{v}^{*} \in \operatorname{int} C, \mathbf{v}^{*}$ must also be a solution of minimizing the objective function in (94) over the set $\mathrm{V} \cap \mathbf{B}_{\rho}$ and $\left(\mathbf{v}^{*}, \zeta^{*}\right)$ must be a solution of $\mathrm{VI}\left(F,\left(\mathrm{~V} \cap \mathbf{B}_{\rho}\right) \times S\right)$, i.e., VI (66) defined on $\left(\mathrm{V} \cap \mathbf{B}_{\rho}\right) \times S$. Finally, applying theorem 4.2 in Kinderlehrer and Stampacchia (1980) (chapter 1, page $13), \mathbf{v}^{*}$ is also a solution of (VI) in (66).

Note that for congested models without sharp capacity bounds, it is not necessary that special paths exist, similar to those described in assumption 3.2, and only continuity and positivity are required for functions $t_{a}(\cdot), a \in A, \sigma_{a}(\cdot), a \in \hat{A}$, on the space of acyclic path flows $\hat{\mathrm{V}}$.

\subsection{Models with explicit capacity bounds}

Generally, functions modeling effective frequencies $f_{a}(\cdot)$ at stops depend on total flows on links close to the boarding link $a$. When the C3F model must reflect the strict limitations of vehicle capacities, it seems logical that effective frequencies depend on total flows of links $a, c, b$ and $e$ as shown in figure 2 below and vanish when these flows are such that vehicle capacity is reached.

Figure 2 .

In general, it seems reasonable to consider functional forms of the type:

$$
f_{a}(v)= \begin{cases}\phi_{a}\left(\frac{v_{a}}{\mu c-v_{e}+v_{a}}\right) & \text { if } v_{e}<\mu c \\ 0 & \text { otherwise }\end{cases}
$$

where $\phi_{a}(\cdot)$ is a decreasing function, $c$ is the vehicle capacity and $\mu$ is a factor related to the time horizon of the planning model being used. In general, the ratio $\rho_{a}$ at boarding link $a \in \hat{E}(i), i \in \hat{N}$ is given by

$$
\rho_{a}(v)=\frac{v_{a}}{\mu c-v_{m(a)}}, \quad\left(v_{m(a)} \triangleq v_{e}(a)-v_{a}\right)
$$

and can be interpreted as the loading factor of passenger queues that are boarding segment $e$ at stop node $i$. An explicit capacity limitation would then be $\rho_{a}<1$, or equivalently, $v_{a}<\mu c-v_{m(a)}$. It seems appropriate to use functions $\phi_{a}(\cdot)$ so that $\phi_{a}(1)=0$. For example, Cepeda et al. (2006) consider in their numerical tests efff, for which $\phi_{a}(x)=\mu\left(1-x^{\beta}\right)$.

Motivated by previous considerations, although not limited at all by the functional forms presented for the efff functions, consider now the case of a polyhedron $X$ defined by explicit linear constraints that (possibly) model capacity limits imposed at some links of the network. To avoid further complexities, the case of nonlinear constraints has not been considered.

$$
X \triangleq\left\{\mathbf{u} \in \mathbb{R}^{|D||A|} \mid E \mathbf{u} \leq c\right\}
$$

where matrix $E=\left(\ldots, E_{d}, \ldots ; d \in D\right) \neq(0)$ is composed of block matrixes $E_{d}$. $E$ is a $k \times|D||A|$ matrix with elements $e_{q, a}^{d} \geq 0$ and $c \in \mathbb{R}^{k}, c>0$. Constraints defining set $X$ can then be written as $\sum_{d \in D} \sum_{a \in A} e_{q, a}^{d} u_{a}^{d} \leq c_{j}, 1 \leq q \leq k$ and $\operatorname{int} X=\{\mathbf{u} \in X \mid E \mathbf{u}<c\}$.

Assumption 5.2 We assume that $\hat{\mathrm{V}} \cap$ int $X \neq \emptyset$.

Assumption 5.3 Functions $t_{a}(\cdot), a \in A$ and $\sigma_{a}(\cdot), a \in \hat{E}(i), i \in \hat{N}$ are continuous, positive, and $<+\infty$ on $\mathrm{V} \cap$ int $X$.

Consider $\mathbf{v} \in \operatorname{fr}(\mathrm{V} \cap \operatorname{int} X)$, i.e., a feasible flow $\mathbf{v}$ on the frontier imposed by constraints that define $X$. Then, the following two subsets of links in the expanded network associated with $\mathbf{v}$ will be used in this subsection:

$$
\begin{aligned}
& A_{\sigma}^{\infty}(\mathbf{v}) \triangleq\left\{a \in \hat{A} \mid \sigma_{a}(v)=+\infty\right\} \\
& A_{t}^{\infty}(\mathbf{v}) \triangleq\left\{a \in A \mid t_{a}(v)=+\infty\right\}
\end{aligned}
$$


If assumption 5.2 is verified, then, for $\epsilon, 0 \leq \epsilon \leq \epsilon_{0}$, consider

$$
X_{\epsilon} \triangleq\left\{\mathbf{u} \in \mathbb{R}^{|D||A|} \mid E \mathbf{u} \leq c-\epsilon[1]_{k}\right\}
$$

so that $\mathrm{V} \cap X_{\epsilon}$ is non empty. For convenience, consider functional $F(\cdot)$ defined in $(77)$ in its block components $F(\cdot)=\left(F_{v}(\cdot), F_{\zeta}(\cdot)\right)$.

Proposition 5.4 Assume that $\operatorname{ray}(\mathrm{V}) \neq \emptyset$ (i.e., the expanded transit network contains cycles). Under assumption 5.3, if $\vec{w} \in \overrightarrow{\mathrm{V}}$, then $F_{v}(\mathbf{v}, \zeta)^{\top} \vec{w}>0, \forall(\mathbf{v}, \zeta) \in(\mathrm{V} \cap \operatorname{int} X) \times S$.

Proof: It suffices to notice that rays in $\overrightarrow{\mathrm{V}}$ are made by flows on cycles on the expanded network and the expression for $F_{v}$ given in $(77)$.

Consider now (VI) in (66) but defined on $(\mathrm{V} \cap X) \times S$, and denote it by $\mathrm{VI}(F,(\mathrm{~V} \cap X) \times S), F(\cdot)$ being the functional defined in (77). The following lemma 5.5 is a preliminary result required in the proof of theorem 5.8.

Lemma 5.5 Under assumptions 5.2 and 5.3, assume that for $\epsilon>0, \mathrm{~V} \cap X_{\epsilon} \neq \emptyset$. Then, $\mathrm{VI}\left(F,\left(\mathrm{~V} \cap X_{\epsilon}\right) \times S\right)$ has a nonempty solution set, and if $\left(\mathbf{v}_{\epsilon}^{*}, \zeta_{\epsilon}^{*}\right)$ is one such solution, then $\mathbf{v}_{\epsilon}^{*} \in \hat{\mathrm{V}} \cap X_{\epsilon}$, i.e., it contains no cyclic flows.

Proof: Consider $C=\bigotimes_{d \in D} C_{d}$, where boxes $C_{d}$ are defined as $C_{d}=\left\{\mathbf{v}^{d} \in \mathbb{R}^{|A|} \mid 0 \leq v_{a}^{d} \leq \rho_{d}, a \in A\right\}$. Bounds $\rho_{d}, d \in D$, are taken as $\rho_{d}>\sum_{(p, d) \in W} g_{p, d}$. Then, $\left.\operatorname{VI}\left(F,\left(\mathrm{~V} \cap X_{\epsilon} \cap C\right) \times S\right)\right)$ has a solution $x_{\epsilon}^{*}=\left(\mathbf{v}_{\epsilon}^{*}, \zeta_{\epsilon}^{*}\right)$ because it is defined on a compact and convex set and because $\mathrm{V} \cap X_{\epsilon} \subset \mathrm{V} \cap$ int $X, F$ is continuous on $\mathrm{V} \cap X_{\epsilon}$. Now, the following equalities must hold:

$$
\begin{array}{ccccc}
\operatorname{Min} & \mathbf{u}^{\top} F_{v}\left(x_{\epsilon}^{*}\right) & \stackrel{(1)}{=} & \operatorname{Min} \underset{\mathbf{u}}{\mathbf{u}^{\top} F_{v}\left(x_{\epsilon}^{*}\right)-\mu^{\top}\left(c_{\epsilon}-E \mathbf{u}\right)} \stackrel{\stackrel{(2)}{=}}{\operatorname{Min}} \underset{\mathbf{u} \in \mathrm{V} \cap C}{X_{\epsilon} \cap C} \quad \mathbf{u}^{\top} F_{v}\left(x_{\epsilon}^{*}\right) \\
\mathbf{u} \in \mathrm{V} \cap X_{\epsilon}
\end{array}
$$

where $c_{\epsilon}=c-\epsilon[1]_{k}$. Equality (1) follows by lagrangian duality. There must exist multipliers $\mu \geq 0$ such that the second linear program in (101) has solutions verifying $\mu^{\top}\left(c_{\epsilon}-E \mathbf{u}\right)=0$ and $E \mathbf{u} \leq c_{\epsilon}$ and that also solve the first linear program in (101). These solutions must lie in $\hat{V}$ because, as a consequence of proposition 5.4, costs of the second linear program are $F_{v}\left(x_{\epsilon}^{*}\right)+E^{\top} \mu>0$ and thus the solutions lie in $\hat{\mathrm{V}} \cap X_{\epsilon} \cap C$. Equality (2) follows because bounds $\rho_{d}$ will never be reached in a solution of the second linear program in (101), and consequently, they will never be reached in solutions of the first.

Primal gap function $G_{P}$ for problem $\mathrm{VI}\left(F,\left(\mathrm{~V} \cap X_{\epsilon}\right) \times S\right)$ will also verify theorem 4.9 (simply consider $\mathrm{V} \cap X_{\epsilon}$ instead of $\mathrm{V}$ in theorem 4.9). If functions $t_{a}(\cdot)$ and inverse efff's $\sigma_{a}(\cdot)$ are continuous and finite on $\hat{\mathrm{V}} \cap X$, then theorem 4.9 will also apply for problem $\mathrm{VI}(F,(\mathrm{~V} \cap X) \times S)$. For these two problems, the Cepeda, Cominetti and Florian gap function will be denoted by $\hat{G}_{\mathrm{CCF}}^{\epsilon}(\cdot)$ and $\hat{G}_{\mathrm{CCF}}(\cdot)$, respectively. It should be noted that the gap function $\hat{G}_{\mathrm{CCF}}(\cdot)$ defined below in (103) for the capacitated case is of special algorithmic interest.

The following relationships will be verified:

$$
\begin{array}{r}
\hat{G}_{\mathrm{CCF}}(\mathbf{v})=\varphi(\mathbf{v}, v)-\operatorname{Min} \mathbf{u} \in \operatorname{V} \cap X \varphi(\mathbf{u}, v), \quad \hat{G}_{\mathrm{CCF}}^{\epsilon}(\mathbf{v})=\varphi(\mathbf{v}, v)-\operatorname{Min} \mathbf{u} \in \operatorname{V} \cap X_{\epsilon} \varphi(\mathbf{u}, v) \\
\hat{G}_{\mathrm{CCF}}(\mathbf{v})=\hat{G}_{\mathrm{CCF}}^{(0}(\mathbf{v})-\hat{G}_{\mathrm{CCF}}^{(1}(v), \quad \hat{G}_{\mathrm{CCF}}^{\epsilon}(\mathbf{v})=\hat{G}_{\mathrm{CCF}}^{(0, \epsilon}(\mathbf{v})-\hat{G}_{\mathrm{CCF}}^{(1, \epsilon}(v)
\end{array}
$$

where $\hat{G}_{\mathrm{CCF}}^{(0}(\mathbf{v})=\hat{G}_{\mathrm{CCF}}^{(0, \epsilon}(\mathbf{v})=G_{\mathrm{CCF}}^{(0}(\mathbf{v})=\varphi(\mathbf{v}, v)$, and

$$
\hat{G}_{\mathrm{CCF}}^{(1}(v)=\operatorname{Min} \mathbf{u} \in \mathrm{V} \cap X \varphi(\mathbf{u}, v), \quad \hat{G}_{\mathrm{CCF}}^{(1, \epsilon}(v)=\operatorname{Min} \mathbf{u} \in \operatorname{V} \cap X_{\epsilon} \varphi(\mathbf{u}, v)
$$

Note that $\mathrm{g}(\epsilon) \triangleq \hat{G}_{\mathrm{CCF}}^{(1, \epsilon}\left(v_{\epsilon}\right)$ is a continuous function near 0 and that the point-to-set map $\epsilon \mapsto$ Sol $\mathrm{VI}\left(F,\left(\mathrm{~V} \cap X_{\epsilon}\right)\right) \times S$ is upper semi-continuous near 0 (as a direct consequence of theorem 2.2.2, page 14, in Fiacco (1983)). Because of lemma 5.5, solution flows $\mathbf{v}^{*}$ of $\mathrm{VI}\left(F,\left(\mathrm{~V} \cap X_{\epsilon}\right) \times S\right)$ are such that $\mathbf{v}^{*} \in \hat{\mathrm{V}}$, and thus an analysis based on acyclic paths, which is necessary for subsequent results in this subsection, can be made. Consider $\Gamma_{\omega}$, the set of acyclic paths joining origin-destination pair $\omega \in W$ on the expanded transit network, and let $H_{\omega}$ be the polytope of the acyclic path flows joining origin-destination pair $\omega \in W: H_{\omega} \triangleq\left\{\mathrm{h} \in \mathbb{R}_{+}^{\left|\Gamma_{\omega}\right|} \mid \sum_{r \in \Gamma_{\omega}} h_{r}^{\omega}=g_{\omega}\right\}$. 
Correspondingly, let $H_{d}, d \in D$ and $H$ be defined as $H_{d} \triangleq \bigotimes_{\omega \in W_{d}} H_{\omega}, \quad d \in D$ and $H \triangleq \bigotimes_{\omega \in W} H_{\omega}$. Let $\Delta=\left(\ldots, \Delta_{d}, \ldots ; d \in D\right)$ be the link path incidence matrix for the expanded transit network composed of block matrices $\Delta_{d}$ with columns associated with paths with common destination $d \in D$. Let also $\mathrm{h}=$ $\left(\ldots, \mathrm{h}_{d}, \ldots ; d \in D\right)$ be a path flow vector with block components $\mathrm{h}_{d}$ for flows on paths and $\mathrm{T}^{d}\left(v, \zeta^{d}\right)$ be the link-cost vector defined in (58) with common destination $d \in D$ and $\mathrm{T}(v, \zeta)=\left(\ldots, \mathrm{T}^{d}\left(v, \zeta^{d}\right), \ldots ; d \in D\right)$. Let the linear application $\mathrm{w}(\cdot): \mathrm{V} \rightarrow \mathcal{V} \subset \mathbb{R}^{|A|}$ be defined as $\mathrm{w}(\mathbf{v})=\sum_{d \in D} \mathbf{v}^{d}$ i.e., $\mathrm{w}(\cdot)$ defines total link flows $v$ for per-destination flow vectors $\mathbf{v}^{d}, d \in D$. Per-destination flow vector $\mathbf{v}^{d}$ will be expressed as $\mathbf{v}^{d}=\Delta_{d} \mathrm{~h}_{d}$, and a path-cost operator will be defined as $\mathrm{J}(\mathrm{h}, \zeta) \triangleq \Delta^{\top} \mathrm{T}(\mathrm{w}(\Delta \mathrm{h}), \zeta)$, where component $C_{\gamma}$ of this operator for path $\gamma$ in the set of paths $\Gamma_{\omega}$, for origin-destination pair $\omega=(i, d) \in W$ is expressed as $J_{\gamma}(\mathrm{h}, \zeta)=C_{\gamma}(\mathrm{w}(\Delta \mathrm{h}), \zeta)$, being:

$$
C_{\gamma}\left(v, \zeta^{d}\right)=\sum_{a \in \gamma} \Psi_{a}^{d}\left(v, \zeta^{d}\right), \quad \gamma \in \Gamma_{\omega}, \omega \in W
$$

Then, variational inequality $\mathrm{VI}(F,(\mathrm{~V} \cap X) \times S)$ or $\mathrm{VI}\left(F,\left(\mathrm{~V} \cap X_{\epsilon}\right) \times S\right)$ can be expressed in terms of path costs and path flows on $H_{X} \triangleq\{\mathrm{h} \in H \mid E \Delta \mathrm{h} \leq c\}$ and $H_{X}^{\epsilon} \triangleq\left\{\mathrm{h} \in H \mid E \Delta \mathrm{h} \leq c-\epsilon[1]_{k}\right\}$ as

$$
\Delta_{d}^{\top} \mathrm{T}^{d}\left(\mathrm{w}(\Delta \mathrm{h}), \zeta^{d}\right)=\Lambda^{d}+\Delta_{d}^{\top} \xi^{d}-\Delta_{d}^{\top} E_{d} \tilde{\theta}
$$

with complementarities $\mathrm{h}_{d} \geq 0, \Delta_{d}^{\top} \xi^{d} \geq 0$ and $\mathrm{h}_{d}^{\top} \Delta_{d}^{\top} \xi^{d}=0$, which are a consequence of complementarities $\mathbf{v}^{d} \geq 0, \xi^{d} \geq 0, \mathbf{v}^{d \top} \xi^{d}=0$. In turn, variables $\zeta^{d}=\left(\ldots, \zeta_{a}^{d}, \ldots ; a \in \hat{E}(i), i \in \hat{N}_{d}\right)$ are solutions of V.I.'s (65) with $\mathbf{v}$ taken as $\Delta \mathrm{h}$ instead. The vector of multipliers $\Lambda^{d}, d \in D$ in (106) is defined as $\Lambda^{d}=\left(\ldots, \lambda_{i}^{d}, \ldots ;(i, d) \in W\right)$ and $\tilde{\theta} \in \mathbb{R}^{k}$ is a non-negative vector of multipliers for constraints $E \Delta \mathrm{h} \leq c$ or $E \Delta \mathrm{h} \leq c-\epsilon[1]_{k}$.

Assumption 5.6 For any $\omega \in W$, there exists at least a path $\gamma_{\omega}$ so that any link a $\in \gamma_{\omega}$ verifies that travel time functions $t_{a}(\cdot), a \in \gamma_{\omega}$, and efff's $\sigma_{a}(\cdot), a \in \hat{A} \cap \gamma_{\omega}$ are continuous and finite on $\hat{\mathrm{V}} \cap X$.

Assumption 5.7 For any $\mathbf{v} \in \operatorname{fr}(\mathrm{V} \cap \operatorname{int} X), A_{t}^{\infty}(\mathbf{v}) \neq \emptyset$ and $/$ or $A_{\sigma}^{\infty}(\mathbf{v}) \neq \emptyset$.

Theorem 5.8 Under assumptions 5.2, 5.3, 5.6, 5.7, consider problem $\mathrm{VI}(F,(\mathrm{~V} \cap X) \times S)$.

1. If there exists a path $\gamma$ from node $i$ to destination $d \in D$ comprised of links that are not included in constraints $E \mathbf{u} \leq c$ defining set $X$, i.e., for any link $a \in \gamma, e_{q, a}^{d}=0,1 \leq q \leq k$, then at a solution $\mathbf{v}^{*}$

$$
\lambda_{i}^{d} \leq C_{\gamma}\left(v^{*}, \zeta^{d *}\right) \leq \sum_{a \in \gamma}\left(t_{a}\left(v^{*}\right)+\tilde{\sigma}_{a}\left(v^{*}\right)\right)
$$

and origin-destination travel time $i \rightarrow d$ is finite.

2. Then, at solutions $\mathbf{v}^{*}$ of problem $\mathrm{VI}(F,(\mathrm{~V} \cap X) \times S)$, function $\hat{G}_{\mathrm{CCF}}^{(1}$ verifies

$$
\hat{G}_{\mathrm{CCF}}^{(1}\left(\mathbf{v}^{*}\right) \leq \sum_{\omega \in W} g_{\omega} C_{\gamma_{\omega}}\left(v^{*}, \zeta^{d(\omega) *}\right) \leq \sum_{\omega \in W} g_{\omega}\left(\sum_{a \in \gamma_{\omega}}\left(t_{a}\left(v^{*}\right)+\tilde{\sigma}_{a}\left(v^{*}\right)\right)\right)
$$

where $d(\omega)$ is the destination of origin-destination pair $\omega \in W$ and $\tilde{\sigma}_{a}(v)$ is defined as $\tilde{\sigma}_{a}(v)=\sigma(v)$ if $a \in \hat{A}$ and otherwise $\tilde{\sigma}_{a}(v) \equiv 0$.

3. Problem $\mathrm{VI}(F,(\mathrm{~V} \cap X) \times S)$ has a nonempty solution set, and any solution $x^{*}=\left(\mathbf{v}^{*}, \zeta^{*}\right)$ is such that $\mathbf{v}^{*} \in \hat{\mathrm{V}} \cap$ int $X$. In other words, solution flows of $\mathrm{VI}(F,(\mathrm{~V} \cap X) \times S)$ contain no cyclic flows on the expanded graph and $E \mathbf{v}^{*}<c$.

In addition, if previous assumptions 5.6, 5.7 hold, then origin-destination travel times are always finite, independently of the level of demands $g_{\omega}, \omega \in W$.

Proof: Let $\mathbf{v}_{\epsilon}^{d *}, d \in D$ be a solution of problem $\mathrm{VI}\left(F,\left(\mathrm{~V} \cap X_{\epsilon}\right) \times S\right)$. Because of lemma $5.5, \mathbf{v}_{\epsilon}^{*} \in \hat{\mathrm{V}}$. Let $\mathrm{h}_{d}^{*}$ be path flows in $H_{X}^{\epsilon}$ such that $\mathbf{v}_{\epsilon}^{d *}=\Delta_{d} \mathrm{~h}_{d}^{*}$ and let $v^{*}=\mathrm{w}\left(\Delta \mathrm{h}^{*}\right)$. Then, function $\hat{G}_{\mathrm{CCF}}^{(1, \epsilon}$ at $\mathbf{v}_{\epsilon}^{*}$ for this problem can be expressed as:

$$
\hat{G}_{\mathrm{CCF}}^{(1, \epsilon}\left(\mathbf{v}_{\epsilon}^{*}\right)=\sum_{d \in D} \mathrm{~T}^{d}\left(v_{\epsilon}^{*}, \zeta_{\epsilon}^{d *}\right)^{\top} \Delta_{d} \mathrm{~h}_{d}^{*}=\sum_{d \in D} \mathrm{~h}_{d}^{* \top} \Delta_{d}^{\top} \mathrm{T}^{d}\left(v_{\epsilon}^{*}, \zeta_{\epsilon}^{d *}\right)
$$


Thus, (106) will hold at solution $\mathrm{h}^{*}$ of problem $\mathrm{VI}\left(F,\left(\mathrm{~V} \cap X_{\epsilon}\right) \times S\right)$ and for path $\gamma \in \Gamma_{\omega}$ referred to in point 1 of the lemma $E_{d} \Delta_{\gamma}=0$, and:

$$
\lambda_{i}^{d}=\min _{\gamma^{\prime} \in \Gamma_{\omega}}\left\{C_{\gamma^{\prime}}^{\omega}\left(v_{\epsilon}^{*}, \zeta_{\epsilon}^{d(\omega) *}\right)+\Delta_{\gamma^{\prime}}^{\top} E_{d}^{\top} \tilde{\theta}\right\} \leq \sum_{a \in \gamma}\left(t_{a}\left(v_{\epsilon}^{*}\right)+\tilde{\sigma}_{a}\left(v_{\epsilon}^{*}\right)\right)
$$

Because no link in path $\gamma$ is included in constraints $E \mathbf{v} \leq c$ and $0 \leq \zeta_{a}^{d} \leq 1$, inequality in (107) follows for flows $v_{\epsilon}^{*}$. Now, because of (106) and complementarities,

$$
\mathrm{h}_{d}^{* \top} \Delta_{d}^{\top} \mathrm{T}^{d}\left(v_{\epsilon}^{*}, \zeta_{\epsilon}^{d *}\right)=\mathrm{h}_{d}^{* \top} \Lambda^{d}-\mathrm{h}_{d}^{* \top} \Delta_{d}^{\top} E_{d} \tilde{\theta} \leq \mathrm{h}_{d}^{* \top} \Lambda^{d}
$$

Because $\hat{G}_{\mathrm{CCF}}^{(1, \epsilon}\left(\mathbf{v}_{\epsilon}^{*}\right)$ can be expressed as in (109), summing for $d \in D$ in (111) there follows (108) for function $\hat{G}_{\mathrm{CCF}}^{(1, \epsilon}$ at $\mathbf{v}_{\epsilon}^{*}$.

At equilibrium solutions, function $\mathrm{g}(\epsilon) \triangleq \hat{G}_{\mathrm{CCF}}^{(1, \epsilon}\left(\mathbf{v}_{\epsilon}^{*}\right)=G_{\mathrm{CCF}}^{(0}\left(\mathbf{v}_{\epsilon}^{*}\right)$ is continuous. If it is taken into account that functions $G_{\mathrm{CCF}}^{(0}$ and functions $t_{a}(\cdot), \sigma_{a}(\cdot)$ are continuous on $\mathrm{V} \cap \operatorname{int} X$ and also that the point-to-set map $\epsilon \mapsto \operatorname{Sol} \operatorname{VI}\left(F,\left(\mathrm{~V} \cap X_{\epsilon}\right)\right) \times S$ is upper semi-continuous near 0 , it follows that,

$$
\lim _{\epsilon \rightarrow 0+} G_{\mathrm{CCF}}^{(1, \epsilon}\left(\mathbf{v}_{\epsilon}^{*}\right)=G_{\mathrm{CCF}}^{(0}\left(\mathbf{v}_{0+}^{*}\right) \leq \sum_{\omega \in W} g_{\omega}\left(\sum_{a \in \gamma_{\omega}}\left(t_{a}\left(v_{0+}^{*}\right)+\tilde{\sigma}_{a}\left(v_{0+}^{*}\right)\right)\right)<+\infty
$$

and results 1 and 2 in theorem follow. Because $A_{t}^{\infty}(\mathbf{v}) \neq \emptyset$ and/or $A_{\sigma}^{\infty}(\mathbf{v}) \neq \emptyset$, then all functions $t_{a}(\cdot)$ and $\sigma_{a}(\cdot)$ are finite at $\mathbf{v}_{0+}^{*} \notin \operatorname{fr}(\mathrm{V} \cap \operatorname{int} X)$.

Let $\rho^{\prime}>\max _{u \in \operatorname{vertex}(\mathrm{V})}\|u\|_{2}$ and $\rho=\left(\rho^{\prime 2}+\sum_{d \in D}\left|\hat{N}_{d}\right|\right)^{1 / 2}$. Because of lemma $5.5, \mathbf{v}_{\epsilon}^{*} \in \hat{\mathrm{V}} \cap \operatorname{int} X$ and $\left\|\mathbf{v}_{\epsilon}^{*}\right\|_{2}<\rho$. Then,

$$
\mathbf{v}_{\epsilon}^{* \top} F_{v}\left(x_{\epsilon}^{*}\right)=\underset{\mathbf{u} \in \mathrm{V} \cap X_{\epsilon}}{\mathbf{u}^{\top} F_{v}\left(x_{\epsilon}^{*}\right)}=\underset{\mathbf{u} \in \mathrm{V}}{\operatorname{Min}} \mathbf{u}^{\top} F_{v}\left(x_{\epsilon}^{*}\right)=\underset{\mathbf{u} \in \mathrm{V} \cap B_{\rho^{\prime}}}{\mathbf{u}^{\top} F_{v}\left(x_{\epsilon}^{*}\right)}
$$

Because of (113) for any sequence $\left\{\epsilon_{\ell}\right\} \rightarrow 0+$, its associated sequence of solutions $\left\{x_{\epsilon_{\ell}}\right\}$ will have a limit point $x_{0+}=\left(\mathbf{v}_{0+}, \zeta_{0+}\right) \in \operatorname{Sol} \mathrm{VI}\left(F,((\mathrm{~V} \cap X) \times S) \cap \mathbb{B}_{\rho}\right)$ with $\mathbf{v}_{0+}^{*} \in \hat{\mathrm{V}}$, and as a consequence, $\left\|x_{0+}\right\|_{2}<\rho$. Applying theorem 4.2 in Kinderlehrer and Stampacchia (1980) (chapter 1, page 13), it follows that $\left.x_{0+} \in \operatorname{Sol} \mathrm{VI}(F,(\mathrm{~V} \cap X) \times S)\right)$ and $\mathbf{v}_{0+} \in \hat{\mathrm{V}} \cap \operatorname{int} X$.

Remark 5.9 Note that, as stated in subsection 4.1, finite inverse efff's were guaranteed by assumption 3.2 and that positive efff's in the solution were conjectured to state the presented reformulation as V.I. of the C3F model. Now, under conditions for either theorem 5.1 or theorem $5.8, f_{a}(v)=1 / \sigma_{a}(v)>0$. Therefore assumption 3.2 can be replaced by those required in theorem 5.1 or theorem 5.8 according to whether the model has no sharp capacity constraints or, on the contrary, these constraints appear in the model.

Finally, taking into account theorem 5.1 and theorem 5.8 and previous remark 5.9, theorem 4.6 may be expressed as one of the following two:

Theorem 5.10 If $t_{a}(\cdot), a \in A$ and $\sigma_{a}(\cdot), a \in \hat{A}$ are continuous and positive on $\mathrm{V}$, then equilibrium flows $\mathbf{v}^{*}$ for the C3F model exist such that $\mathbf{v}^{*} \in \hat{\mathrm{V}}$, and also solve $\mathrm{VI}(F, \mathrm{~V})$. The converse is also true and thus C3F model is equivalent to (VI) in (66), $\mathrm{VI}(F, \mathrm{~V})$.

Theorem 5.11 If (explicit or implicit) sharp capacity constraints defining $X$ as in (97) are such that $\hat{\mathrm{V}} \cap \operatorname{int} X \neq \emptyset$, then

1. Suppose that travel time functions $t_{a}(\cdot), a \in A$, and efff's $\sigma_{a}(\cdot), a \in \hat{A}$ are positive and continuous on $\mathrm{V} \cap X$. Then, any solution $\mathbf{v}^{*}$ of $\mathrm{VI}(F, \mathrm{~V} \cap X)$ is such that $\mathbf{v}^{*} \in \hat{\mathrm{V}} \cap X$.

2. Suppose that travel time functions $t_{a}(\cdot), a \in A$, and efff's $\sigma_{a}(\cdot), a \in \hat{A}$ are positive and continuous on $\mathrm{V} \cap \operatorname{int} X$ and that assumptions 5.6 and 5.7 hold. Then, equilibrium flows $\mathbf{v}^{*}$ for the C3F model exist and are such that $\mathbf{v}^{*} \in \hat{\mathrm{V}} \cap$ int $X$.

In both cases origin-destination travel times are finite independently of the demands $g_{\omega}, \omega \in W$. 
The following corollary is also a direct consequence of theorem 5.11 and lemma 4.8

Corollary 5.12 ( Characterization of C3F equilibrium as a fixed-point inclusion). Under conditions of theorem 5.11, $\mathbf{v}^{*}$ is a solution of $\mathrm{VI}(F, \mathrm{~V} \cap X)$ iff the following fixed-point inclusion is verified:

$$
\mathbf{v}^{*} \in \operatorname{Sol} \operatorname{Min} \mathbf{u} \in \operatorname{V} \cap X \varphi\left(\mathbf{u}, \mathrm{w}\left(\mathbf{v}^{*}\right)\right)
$$

where $\mathrm{w}\left(\mathbf{v}^{*}\right)=\sum_{d \in D} \mathbf{v}^{d *}$.

For explicitly capacitated transit networks, result 3 in theorem 5.8 cannot be obtained by simply imposing assumptions such as 5.7 on functions $\sigma_{a}(\cdot)$ and the stronger one in 5.6 is required, as the following simple example shows.

Consider the small network shown in figure 3. In-vehicle times for lines 1 and 2 from A to $\mathrm{C}$ are 18 and 20, respectively. Boarding and alighting links are assigned a travel time function $t_{a}=1$. Effective frequency functions for line 1 and 2 at stop node $\mathrm{A}$ are given by:

$$
\begin{aligned}
& f_{1}\left(v_{1}\right)= \begin{cases}1 / 5 & \text { for } v_{1} \leq 60 \\
\max \left\{1 / 5-0.1\left(v_{1}-60\right)^{2}, 0\right\} & \text { for } v_{1} \geq 60\end{cases} \\
& f_{2}\left(v_{2}\right)= \begin{cases}1 / 6 & \text { for } v_{2} \leq 60 \\
\max \left\{1 / 6-\left(v_{2}-60\right)^{2}, 0\right\} & \text { for } v_{2} \geq 60\end{cases}
\end{aligned}
$$

It is assumed that boardings to line 2 at $\mathrm{B}$ experience the same efff $f_{2}$ as those at A. Origin-destination flows are $g_{A C}=100$ and $g_{B C}=\frac{160}{11}+\frac{1}{\sqrt{6}}$. Capacities imposed implicitly by efff's are $\sqrt{2}+60$ for line 1 and $60+1 / \sqrt{6}$ for line 2 . Note, that there is enough capacity on the network to allocate the demand. After performing a congested transit assignment on the example network, average travel time from A to $\mathrm{C}$ is $\frac{260}{11}$, and boardings at node $\mathrm{A}$ on lines 1 and 2 are $v_{1}=\frac{600}{11}$, and $v_{2}=\frac{500}{11}$, respectively. Boardings at node $\mathrm{B}$ are $\frac{160}{11}+\frac{1}{\sqrt{6}}$ and average travel time from $\mathrm{B}$ to $\mathrm{C}$ is $\infty$. Note that all efff's remain uncongested. Note also that adding a new link from $\mathrm{B}$ to $\mathrm{C}$ with a continuous, increasing and finite travel time function would guarantee that origin-destination travel time from $\mathrm{B}$ to $\mathrm{C}$ would always remain finite by absorbing part of the flow $B \rightarrow C$, which now can only pass through line 2 to its destination.

Figure 3 .

\section{Conclusions and further research}

In this paper, it has been proved that the equilibrium model for congested transit networks developed by Cominetti and Correa (2001) and Cepeda et al. (2006) referred to in this paper as the C3F model, can be formulated as a variational inequality problem. Previously, Cepeda et al. (2006) reformulated this equilibrium model as the minimization of a non-convex and non-differentiable gap function and solved it heuristically by means of the classical mean successive averages method. For proof of the equivalent formulation in variational inequalities of the C3F model, partial results in Cepeda et al. (2006) have been used. Conditions for the existence of results of the new VI reformulation of the C3F model are developed in this paper, and the case of a strict capacity constrained congested transit model is examined. It is proved that, under the assumptions shown in this paper, solutions exist and that transit volumes will remain under capacity, avoiding infinite origin-destination travel times and completely saturated links. Furthermore, it is shown that for this case, it is also possible to consider a gap function for the problem that has been derived in a natural way from the one derived in Cepeda et al. (2006) for the uncapacitated case. The contribution in this paper opens the door to the wide spectrum of algorithmic methods for variational inequality problems in order to solve the C3F model, and an in-depth exploration of the algorithmic alternatives opened by this reformulation in variational inequalities is a task that will be developed in a forthcoming paper.

Acknowledgments. Special thanks to the task of two anonymous referees who have contributed to improve the quality and presentation of this paper.

\section{References}


C. Chriqui and P. Robillard, "Common bus lines", Transportation Science 9, 115-121 (1975).

M. Cepeda, R. Cominetti and M. Florian, "A frequency-based assignment model for congested transit networks with strict capacity constraints : characterization and computation of equilibria", Transportation Research B 40 437-459 (2006).

R. Cominetti and J. Correa, "Common-lines and passenger assignment in congested transit networks", Transportation Science 35 (3) 250-267 (2001).

J. De Cea and E. Fernández, "Transit assignment for congested public transport systems: an equilibrium model", Transportation Science, 27 (2) 133-147. (1993).

A. Fiacco, Introduction to Sensitivity and Stability Analysis in Non Linear Programming. Academic Press 1983.

C.J. Goh and X.Q. Yang Duality in optimization and variational inequalities. Optimization Theory and Applications Series, Taylor \& Francis,(2002)

D. Kinderlehrer and G. Stampacchia, An introduction to variational inequalities and their applications. Academic Press, New York, 1980

P. Marcotte, "Advantages and drawbacks of variational inequality formulations" in Variational Inequalities and Network Equilibrium Problems, F. Giannessi and A. Maugeri (eds), 179i-194, Plenum Press 1995.

S. Nguyen and S. Pallotino, "Equilibrium traffic assignment in large scale transit networks", European Journal of Operational Research 37 (2) 176-186 (1988).

T. Rockafellar, Convex Analysis. Princeton University Press (1972). 2nd Edition

M.J. Smith, "Existence, Uniqueness and Stability of Traffic Equilibria", Transportation Research 13B(4) 295-304 (1979).

H. Spiess, Contribution à la théorie et aux outils de planification des réseaux de transport urbains. Ph D thesis, Département d'Informatique et Récherche Opérationnelle, Publication 382, CRT, U. de Montréal (1984)

H. Spiess and M. Florian, "Optimal strategies: a new assignment model for transit networks", Transportation Research Part B 23 (2) 83-102 (1989) 
Figure 1. The transit expanded network assumed in the model.

Figure 2. Links whose total flows intervene in the efff $f_{a}(\cdot)$ for boarding link $a$.

Figure 3. An example showing that an apparently sufficient capacitated network for which assumption 5.6 does not hold may have completely saturated links and $\infty$ origin-destination travel times. 

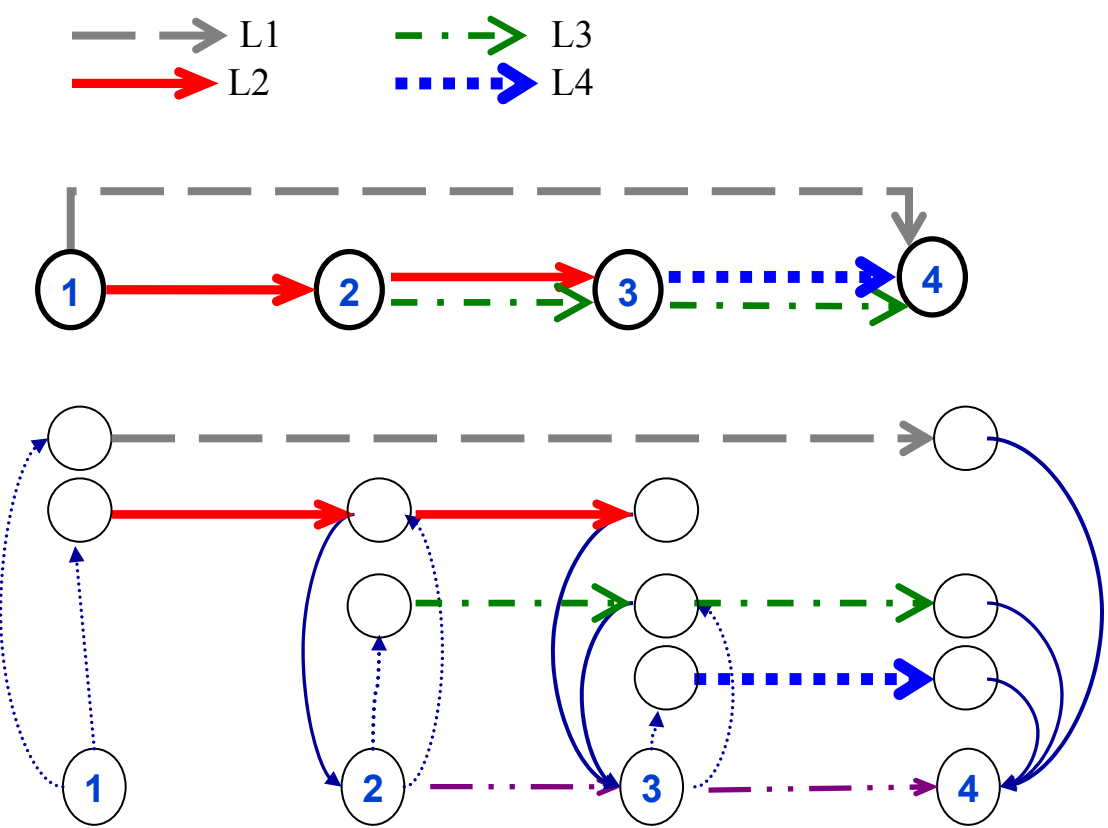

$\longrightarrow$ alighting links $\left(f_{a}=\infty\right)$

b............. boarding links (finite frequency, $f_{a}<\infty$ )

$-\cdots->\quad$ transfer links $\left(f_{a}=\infty\right)$

Figure 1: The transit expanded network assumed in the model. 


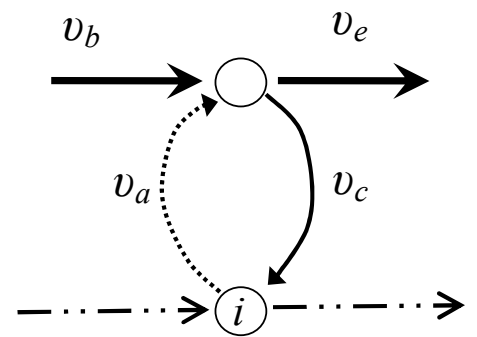

Figure 2: Links whose total flows intervene in the efff $f_{a}(\cdot)$ for boarding link $a$. 
Line 1

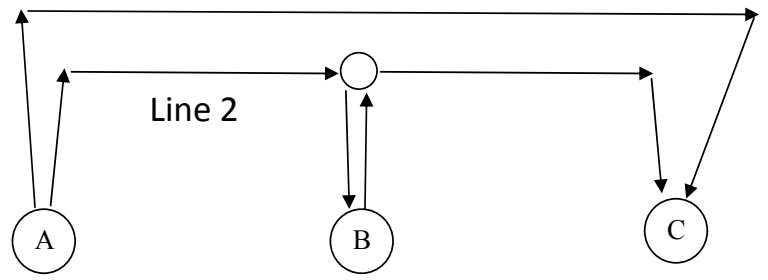

Figure 3: An example showing that an apparently sufficient capacitated network for which assumption 5.6 does not hold may have completely saturated links and $\infty$ origin-destination travel times. 